Pacific Journal of Mathematics

ON THE MAXIMUM DIFFERENCE BETWEEN THE
EMPIRICAL AND EXPECTED HISTOGRAMS FOR SUMS 


\title{
ON THE MAXIMUM DIFFERENCE BETWEEN THE EMPIRICAL AND EXPECTED HISTOGRAMS FOR SUMS
}

\author{
Persi Diaconis and David Freedman
}

\begin{abstract}
Suppose $S_{n}$ is a sum of $n$ independent and identically distributed random variables with $E\left|X_{1}^{3}\right|<\infty$. If $n$ is large, $S_{n}$ is approximately normal. A histogram of $k$ copies of $S_{n}$ will be close to the normal curve if $k$ is large relative to $\sqrt{n} \log n$. This paper derives the joint distribution of the location and size of the maximum deviation between this histogram and the probability histogram for $S_{n}$. When $k$ is large relative to $\sqrt{n}(\log n)^{3}$, the maximum deviation is taken on at a unique location. The location is normally distributed and independent of the size of the maximum deviation, which has a double-exponential distribution. We construct an example, involving Edgeworth-like expansions, to show the behavior changes if $E\left(X_{1}^{2}\right)<\infty$ but $E\left|X_{1}^{3}\right|=\infty$.
\end{abstract}

1. Introduction. The central limit theorem is often used heuristically to justify the approximation of histograms for data by the normal curve. This argument can be made precise through the following model. There is some basic random variable $X$. Take the sum of $n$ independent copies of $X$, and then take $k$ independent copies of these sums. Provided $X$ is well-behaved, $n$ is large, and $k$ is large in relation to $n$, the histogram for the sums will be close to the normal curve. In this way, the model rigorously justifies the use of the normal curve to approximate the data.

In more detail, let $X_{1}, X_{2}, \cdots$ be independent, identically distributed random variables. Suppose the $X_{i}$ are integer-valued and have span 1:

(1.1) g.c.d $\{j-k: j, k \in S>0\}=1$, where $j \in S$ iff $P\left\{X_{1}=j\right\}>0$.

Suppose too

$$
E\left|X_{1}^{3}\right|<\infty \text {. }
$$

Let $S_{n}=X_{1}+\cdots+X_{n}$. Take $k$ independent copies of $S_{n}$, and let $N_{j}$ be the number of these sums which are equal to $j$ : the notation hides the dependence of $N_{j}$ on $n$ and $k$. Up to scaling, the counts $N_{j}$ correspond to the empirical histogram for the $k$ sums. Of course,

$$
E\left(N_{j}\right)=k p_{j}, \text { where } p_{j}=P\left(S_{n}=j\right) .
$$

Up to scaling, the numbers $p_{j}$ correspond to the probability histo- 
gram for the $k$ sums. When $n$ is large, the local central limit theorem implies that the $p_{j}$ are uniformly close to an appropriately scaled normal curve. In this paper, we study the random variable

$$
M_{n k}=\max _{j}\left(N_{j}-k p_{j}\right) \text {. }
$$

Up to scaling, this is the maximum difference between the empirical histogram of the $k$ sums and the probability histogram of the sum. This random variable is the key to understanding the maximum difference between the histogram and the normal curve, as will be shown in a future paper.

We suppose

$$
n \longrightarrow \infty \text { and } k / \sqrt{n}(\log n)^{3} \longrightarrow \infty \text {. }
$$

Assumption (1.5) is discussed at the end of this section. Let

$$
\mu=E\left(X_{1}\right) \text { and } \sigma^{2}=\operatorname{Var} X_{1} .
$$

In Freedman [5] it is shown that if (1.1-1.6) hold, then

$$
M_{n k} / \sqrt{\frac{k}{\sigma \sqrt{2 \pi n}}} \log n \longrightarrow 1 \text { in probability. }
$$

Our main object in this paper is to prove the following result, which sharpens (1.7).

THEOREM 1.8. Assume (1.1-1.6). With probability approaching one, $M_{n k}=\max _{j}\left(N_{j}-k p_{j}\right)$ is taken on at a unique index $L_{n k}$. Moreover, $L_{n k}$ and $M_{n k}$ are asymptotically independent, $L_{n k}$ being asymptotically normal and $M_{n k}$ being asymptotically double-exponential. To be more precise, let

$$
\begin{gathered}
\Phi(y)=\frac{1}{\sqrt{2 \pi}} \int_{-\infty}^{y} \exp \left(-\frac{1}{2} u^{2}\right) d u, \\
z_{n}(x)=\sqrt{\frac{k}{\sigma \sqrt{2 \pi n}}} \cdot \sqrt{\log n-2 \log \log n+x} .
\end{gathered}
$$

Then, the probability that

$$
L_{n k}-n \mu<y \sigma \sqrt{2 n / \log n} \text { and } M_{n k}<z_{n}(x)
$$

converges to

$$
\Phi(y) \exp \left[-\sigma \sqrt{2} e^{-x / 2}\right] .
$$

As usual, $\exp (x)=e^{x}$.

This theorem is illustrated in Table 1 and Figure 1, which report 
TABLE 1

Computer simulation illustrating Theorem (1.8).

\begin{tabular}{|c|c|c|c|c|c|c|c|c|}
\hline \multirow{2}{*}{$n$} & \multirow{2}{*}{$k$} & \multirow{2}{*}{$k /\left[\sqrt{ } \bar{n}(\log n)^{3}\right]$} & lower & quartile & upper & quartile & observed & value \\
\hline & & & $L_{n k}$ & $M_{n k}$ & $L_{n k}$ & $M_{n k}$ & $L_{n k}$ & $M_{n k}$ \\
\hline 25 & 100 & 0.6 & 83 & 3.0 & 92 & 4.9 & 97 & 2.5 \\
\hline 50 & 500 & 1.2 & 169 & 6.2 & 181 & 9.5 & 185 & 8.3 \\
\hline 100 & 10,000 & 10.2 & 341 & 24.9 & 358 & 36.8 & 358 & 26.9 \\
\hline
\end{tabular}

three computer simulations. In all three, the basic random variable $X_{1}$ took the six values 1 through 6 with equal probability $1 / 6$. The table shows the values chosen for $n$ and $k$, the lower and upper quartiles for the asymptotic distributions of $L_{n k}$ and $M_{n k}$, as computed from the theorem, and the values observed in the simulation.

In the first line of Table 1 , the location $L_{n k}$ is reported as 97: so $N_{j}-k p_{j}$ is largest when $j=97$. From the figure, the mode of the empirical histogram is at 82 : so $N_{j}$ is largest when $j=82$. In general, the location of the maximum deviation and the mode are
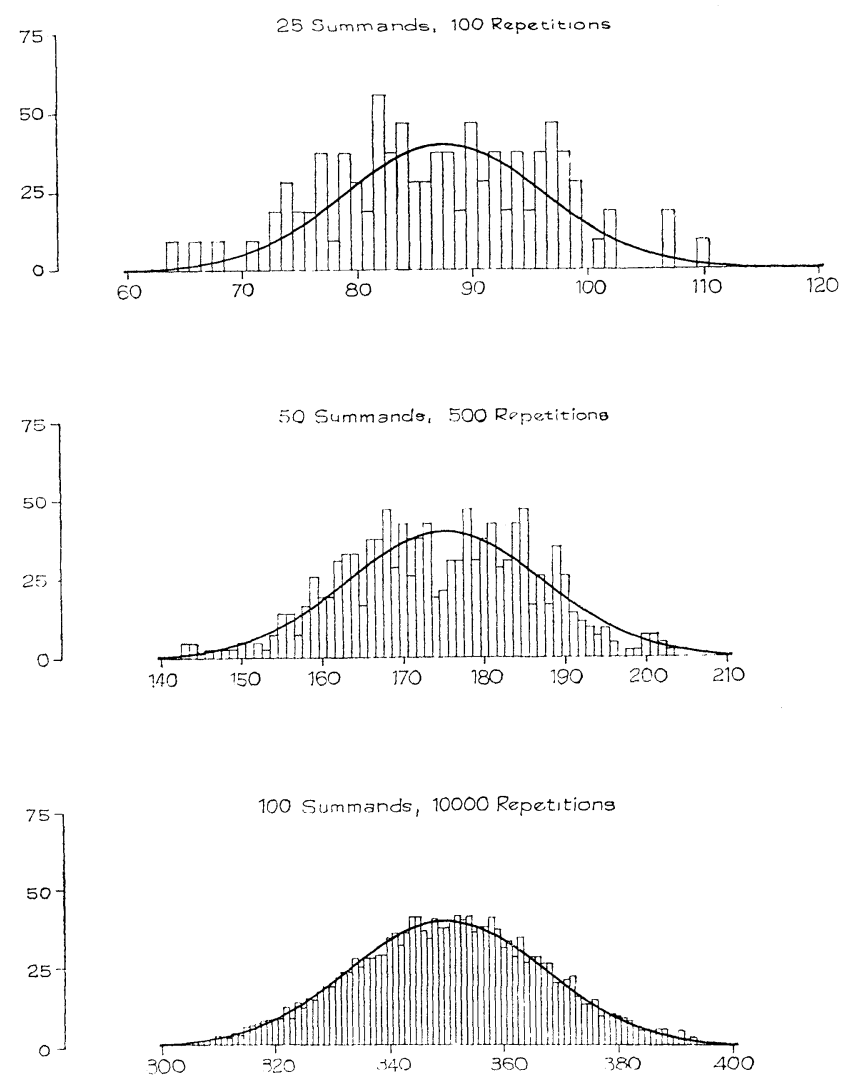

Figure 1

Computer simulation: the empirical histogram converges to the normal curve. 
very different. Compare Theorem (1.8) with [2].

The figure shows the histograms themselves. The horizontal scale shows $j$, but the three axes are arranged so that the means $n \mu$ line up, and the standard deviations $\sigma \sqrt{n}$ cover the same physical distance. The vertical scale shows $(\sigma \sqrt{n}) \times\left(N_{j} / k\right) \times 100 \%$ : that is, $N_{j}$ is converted to a percent (relative to $k$ ), and the histograms are rescaled vertically by $\sigma \sqrt{n}$ to have the same physical area. For a discussion of this convention, see pp. $29 \mathrm{ff}$ and $275 \mathrm{ff}$ of Freedman, Pisani, Purves [6]. Thus, the maximum difference between the rescaled empirical and theoretical histograms is

$$
\frac{\sigma \sqrt{n}}{k} M_{n k}
$$

In the third line of Table 1 , for instance, that is about $5 \%$.

In $\S 2$, we argue heuristically that our problem can be reduced to finding the location and size of the maximum of a sequence $Z_{j}$ of independent, normal variables having mean 0 , but variances $1-\left(j^{2} / 2 \sigma^{2} n\right)$ as $n \rightarrow \infty$. This problem is solved in Proposition (2.2). In $\S 4$, we prove Theorem (1.8). Section 3 gives some technical lemmas, including bounds for the probability of an intersection of events, and approximations for binomial tail probabilities.

In this paper and in Freedman [5] the condition $E\left|X_{1}^{3}\right|<\infty$ was assumed. In fact Lemmas (4.1) and (4.3) of this paper replace Lemma (15) in Freedman [5], and hence prove Theorem (5) of that paper assuming only $E\left(X_{1}^{2}\right)<\infty$.

The main use of the third-moment assumption in this paper is to justify the bound on the error in the local central limit theorem in equation (3.27). In $\S 5$, we construct an example to show that new limiting behavior can occur when $E\left|X_{1}^{3}\right|=\infty$. The argument involves Edgeworth-like corrections to sums of random variables without third moments, and may be of independent interest.

Concerning condition (1.5), if $k \gg \sqrt{n} \log n$ but $k=$ $0\left[\sqrt{n}(\log n)^{3}\right]$, the form of the norming constants in equation (1.10) changes radically, because the large deviations corrections in the normal approximation to the binomial distribution must be accounted for. This is worked out in detail for the closely related problem of the mode of the histogram, in $\S 4$ of Diaconis and Freedman [2]. If $k=0(\sqrt{n} \log n)$, then Freedman [5] shows that the maximum deviation does not converge to zero in probability.

2. A heuristic argument. It is convenient to discuss the behavior of $N_{j}-k p_{j}$ separately for four zones. The zones are 
described in terms of constants $\delta$ and $A$, where $\delta$ is small but positive, $A$ is large but finite. For definiteness, we will assume $0<\delta<1 / 10$. The zones are:

$$
\text { zone I: }|j-n \mu|<\delta \sigma \sqrt{n}
$$

$$
\begin{gathered}
\text { zone II: } \delta \sigma \sqrt{n} \leqq|j-n \mu| \leqq A \sigma \sqrt{n} \\
\text { zone III: } A \sigma \sqrt{n}<|j-n \mu| \leqq \sigma n^{3 / 4} \\
\text { zone IV: } \sigma n^{3 / 4}<|j-n \mu| .
\end{gathered}
$$

As will be shown below, zones II, III, and IV make no contribution to the maximum. In zone I, the $N_{j}-k p_{j}$ are approximately independent and normal, with mean 0 and

$$
\operatorname{Var}\left(N_{j}-k p_{j}\right)=k p_{j}\left(1-p_{j}\right) \doteq k p_{j} \doteq \frac{k}{\sigma \sqrt{2 \pi n}}\left[1-\frac{(j-n \mu)^{2}}{2 \sigma^{2} n}\right] .
$$

Thus, $L_{n k}-n \mu$ and $\sqrt{\sigma \sqrt{2 \pi n} / k} M_{n k}$ should be distributed like the $L_{n}$ and $M_{n}$ of the following proposition.

Proposition 2.2. Let $U_{j}$ be independent normal random variables, with mean 0 and $\operatorname{Var}\left(U_{j}\right)=1-j^{2} / 2 \sigma^{2} n$, for $|j|<\delta \sigma \sqrt{n}$. Let $M_{n}=\max _{j} U_{j}$, and let $L_{n}$ be the index at which the maximum is achieved. Then, as $n$ tends to $\infty$, the probability that

$$
L_{n}<y \sigma \sqrt{2 n / \log n} \text { and } M_{n}<\sqrt{\log n-2 \log \log n+x}
$$

converges to

$$
\Phi(y) \exp \left[-\sigma \sqrt{2} e^{-x / 2}\right] .
$$

As in (1.9), $\Phi$ is the standard normal distribution function.

Proof. For $-\infty \leqq a \leqq b \leqq \infty$, let $I_{a b}$ be the set of $j$ with $|j|<\delta \sigma \sqrt{n}$ and

$$
a \sigma \sqrt{2 n / \log n} \leqq j<b \sigma \sqrt{2 n / \log n} .
$$

Thus, $I_{a b}$ is always finite. Let $M_{a b}$ be the $\max$ of $U_{j}$ for $j \in I_{a b}$. Clearly, if $a<b<c<d$, then $M_{a b}$ and $M_{c d}$ are independent. Abbreviate

$$
w_{n}(x)=\sqrt{\log n-2 \log \log n+x} \text { and } \gamma=\sigma \sqrt{2} .
$$

We will show that

(2.4) $P\left\{M_{a b}<w_{n}(x)\right\} \longrightarrow \exp \left\{-\gamma[\Phi(b)-\Phi(a)] e^{-(1 / 2) x}\right\}$ for $-\infty<a<b<\infty$. 


$$
\begin{aligned}
\limsup _{n \rightarrow \infty} & P\left\{M_{-\infty a}<w_{n}(x)\right\} \\
\quad & \exp \left\{-\gamma \Phi(a) e^{-(1 / 2) x}\right\} \text { for }-\infty<a<0 . \\
\limsup _{n \rightarrow \infty} P\left\{M_{b \infty}<w_{n}(x)\right\} & \leqq \exp \left\{-\gamma[1-\Phi(b)] e^{-(1 / 2) x}\right\} \text { for } 0<b<\infty .
\end{aligned}
$$

Granting (2.4-6), the proposition can be derived by an elementary argument as follows. First, (2.4) holds even for infinite $a$ or $b$, in view of (2.5-6). Now let $Y_{1}$ and $Y_{2}$ be independent double-exponential random variables:

$$
P\left\{Y_{i}<x\right\}=\exp \left[-\gamma_{i} e^{-(1 / 2) x}\right]
$$

where

$$
\gamma_{1}=\gamma \Phi(y) \text { and } \gamma_{2}=\gamma[1-\Phi(y)]
$$

so

$$
\gamma_{1}+\gamma_{2}=\gamma
$$

The function $w_{n}(\cdot)$ defined by $(2.3)$ is continuous and strictly increasing; so is its inverse, $w_{n}^{-1}$. Equation (2.4) implies that the joint distribution of $w_{n}^{-1}\left(M_{-\infty y}\right)$ and $w_{n}^{-1}\left(M_{y \infty}\right)$ converges in distribution to the joint distribution of $Y_{1}$ and $Y_{2}$. Now $P\left(Y_{1}=x\right)=P\left(Y_{1}=Y_{2}\right)=$ 0 . So

$$
\begin{aligned}
P\left\{L_{n}<\right. & \left.y \sigma \sqrt{2 n / \log n} \text { and } M_{n}<w_{n}(x)\right\} \\
= & P\left\{M_{-\infty y}<w_{n}(x) \text { and } M_{-\infty y}>M_{y_{\infty}}\right\} \\
= & P\left\{w_{n}^{-1}\left(M_{-\infty y}\right)<x \text { and } w_{n}^{-1}\left(M_{-\infty y}\right)>w_{n}^{-1}\left(M_{y \infty}\right)\right\} \\
& \longrightarrow P\left\{Y_{1}<x \text { and } Y_{1}>Y_{2}\right\} \\
= & \int_{-\infty}^{\infty} \frac{1}{2} \gamma_{1} e^{-(1 / 2) z} \exp \left[-\gamma_{1} e^{-(1 / 2) z}\right] \exp \left[-\gamma_{2} e^{-(1 / 2) z}\right] d z \\
= & \left.\Phi(y) \int_{-\infty}^{\infty} \frac{1}{2} \gamma e^{-(1 / 2) z} \exp \mid-\gamma^{--(1 / 2) z}\right] d z .
\end{aligned}
$$

The last displayed expression equals

$$
\Phi(y) \exp \left[-\gamma e^{-(1 / 2) x}\right] .
$$

Thus, (2.4-6) imply (2.2).

We turn now to the proof of (2.4). We will write $v_{j}$ for $\operatorname{Var}\left(U_{j}\right)=1-j^{2} / 2 \sigma^{2} n$. Fix an $x$, and abbreviate $w=w_{n}(x)$. In essence, the proof of (2.4) is the follwing computation: 


$$
\begin{aligned}
\log P\left\{M_{a b}<w\right\} & =\sum_{j \in I_{a b}} \log P\left\{U_{j}<w\right\} \\
& \doteq \sum_{j \in I_{a b}} P\left\{U_{j}>w\right\} \\
& \doteq \sum_{j \in I_{a b}} \sqrt{v_{j} / 2 \pi} \cdot \frac{1}{w} \cdot \exp \left\{-\frac{1}{2} w^{2} / v_{j}\right\},
\end{aligned}
$$

by (3.15). The symbol $\doteq$ means "approximately equal" and is only used in heuristic argument. For our purposes

$$
\sqrt{\bar{v}_{j}} / w \doteq 1 / \sqrt{\log n} .
$$

In the exponent, $1 / v_{j} \doteq 1+j^{2} / 2 \sigma^{2} n$. So

(2.10) $\frac{1}{2} w^{2} / v_{j} \doteq \frac{1}{2} \log n-\log \log n+\frac{1}{2} x+\frac{1}{2} j^{2} /\left(2 \sigma^{2} n / \log n\right)$.

Thus, $\log P\left\{M_{a b}<w\right\}$ is approximately $\sqrt{2} \sigma e^{-(1 / 2) x}$ times

$$
\frac{1}{\sqrt{2 \pi}} \frac{1}{\sqrt{2 \sigma^{2} n / \log n}} \sum_{j \in 1_{a b}} \exp \left\{-\frac{1}{2} j^{2} /\left(2 \sigma^{2} n / \log n\right)\right\} .
$$

This last is a Riemann sum for

$$
\frac{1}{\sqrt{2 \pi}} \int_{a}^{b} \exp \left(-\frac{1}{2} u^{2}\right) d u=\Phi(b)-\Phi(a) .
$$

We now indicate the details required to justify (2.8). All " $O$ " and " $o$ " error terms are uniform over $j \in I_{a b}$. Note that $a$ and $b$ are finite, so $j^{2} / n=O(1 / \log n)$. In (2.9), then, the ratio of the two sides converges to one as $n \rightarrow \infty$, uniformly over $j \in I_{a b}$. In (2.10),

$$
\begin{aligned}
\frac{1}{2} w^{2} & \frac{1}{v_{j}}-\frac{1}{2} w^{2}\left(1+\frac{j^{2}}{2 \sigma^{2} n}\right) \\
& =\frac{1}{2} w^{2}\left(\frac{1}{v_{j}}-1-\frac{j^{2}}{2 \sigma^{2} n}\right) \\
& =O(\log n) \cdot o\left(j^{2} / b\right) \\
& =o(1) .
\end{aligned}
$$

So the difference between the two sides of (2.10) is

$$
\begin{aligned}
& \frac{1}{2} w^{2} \frac{j^{2}}{2 \sigma^{2} n}-\frac{1}{2} \log n \cdot \frac{j^{2}}{2 \sigma^{2} n}+o(1) \\
& \quad=\frac{1}{2}\left(w^{2}-\log n\right) \frac{j^{2}}{2 \sigma^{2} n}+o(1) \\
& \quad=O(\log \log n) \cdot O(1 / \log n)+o(1) \\
& \quad=o(1) .
\end{aligned}
$$


Thus, the ratio of $P\left(U_{j}>w\right)$ to $\sqrt{2} \sigma e^{-(1 / 2) x}$ times the $j$ th term in (2.11) goes to one as $n$ goes to infinity, uniformly over $j \in I_{a b}$. As a result,

$$
P\left(U_{j}>w\right)=o(1)
$$

and

$$
\sum_{i \in I_{a b}} P\left(U_{j}>w\right) \longrightarrow \sqrt{2} \sigma e^{-(1 / 2) x}[\Phi(b)-\Phi(a)] .
$$

Now (2.12-13) imply that

$$
\sum_{j \in I_{a b}} \log P\left(U_{j} \leqq w\right)=\sum_{j \in I_{a b}}\left[P\left(U_{j}>w\right)+o\left(P\left(U_{j}>w\right)\right)\right]
$$

also converges to the right side of (2.13).

This completes the proof of (2.4). The proof of (2.5) is very similar. In place of (2.9), we use the estimate

$$
\sqrt{v_{j}} / w \leqq 1 / w .
$$

To modify (2.10), we use the fact that

$$
1 / v_{j} \geqq 1+j^{2} / 2 \sigma^{2} n
$$

so

$$
\frac{1}{2} w^{2} / v_{j} \geqq \frac{1}{2} \log n-\log \log n+\frac{1}{2} x+\frac{1}{2} w^{2} j^{2} / 2 \sigma^{2} n ;
$$

and $\sum_{j \in I-\infty a} P\left\{U_{j}>w\right\}$ is bounded above, to within $o(1)$, by

$$
\begin{aligned}
& \sqrt{2} \sigma e^{-(1 / 2) x} \text { times } w^{-2} \log n \text { times } \\
& \qquad \frac{1}{\sqrt{2 \pi}} \frac{w}{\sqrt{2 \sigma^{2} n}} \sum_{J \in I_{-\infty} a} \exp \left\{-\frac{1}{2} w^{2} j^{2} / 2 \sigma^{2} n\right\} .
\end{aligned}
$$

Now $w^{-2} \log n \rightarrow 1$, and the last display is at most

$$
\Phi(a)+o(1)
$$

because the normal density is monotone increasing on $(-\infty, 0)$. The $o(1)$ corresponds to the term in $j$ at the edge of $I_{-\infty a}$. This completes our discussion of (2.5), and (2.6) is symmetric.

3. Probability approximations. The random variables $N_{j}$ introduced in $\S 1$ have a joint multinomial distribution. We will approximate this distribution by getting upper and lower bounds in terms of independent binomial random variables. The binomial variables are then approximated by an appropriate normal distribution. This section contains the basic bounds and approximations. 
The main results are (3.2), (3.16), and (3.17).

The first lemma is an upper bound for multinomial probabilities due to Mallows [9].

LemmA 3.1. Let $M_{1}, M_{2}, \cdots, M_{J}$ have a joint multinomial distribution. Then, for any real numbers $a_{1}, a_{2}, \cdots, a_{J}$,

$$
P\left(M_{j} \leqq a_{j} \quad \text { for } \quad 1 \leqq j \leqq J\right) \leqq \prod_{j=1}^{J} P\left(M_{j} \leqq a_{j}\right)
$$

The second lemma will be used to get a lower bound for multinomial probabilities.

Proposition 3.2. Let $(\Omega, \mathfrak{F}, P)$ be a probability triple, and let $\widetilde{\mho}_{0} \subset \widetilde{\mho}_{1} \subset \ldots$ be sub- $\sigma$-fields of $\widetilde{\Im}$. Let $A_{j}$ and $G_{j}$ be events in $\widetilde{\mho}_{j}$. Let $\lambda_{j}, \lambda_{j}^{*}$, and $\varepsilon_{j}$ be nonnegative numbers. Suppose $P\left(G_{j}\right) \geqq 1-\varepsilon_{j}$ and

$$
\lambda_{j} \leqq P\left(A_{j} \mid \widetilde{\mho}_{j-1}\right) \leqq \lambda_{j}^{*} \text { on } \quad G_{j-1}
$$

Then $P\left(\bigcap_{j=1}^{r} A_{j}\right)$ is bounded between

$$
\prod_{j=1}^{J} \lambda_{j}-\sum_{j=0}^{J-1} \varepsilon_{j} \text { and } \prod_{J=1}^{J} \lambda_{j}^{*}+\sum_{j=0}^{J-1} \varepsilon_{j} .
$$

Proof. Induction on $J$.

Let $N$ be binomial with parameters $k$ and $p$, where $0<p<1$. The next set of results give approximations for the distribution of $N$ which are uniform as $k$ and $p$ vary over a wide range. Readers may be surprised to find us proving versions of the central limit theorem for coin-tossing, at this late date. However, we need bounds which are uniform as $p$ gets small; to stay in the realm of the central limit theorem, we will require $k p$ to be large. Our results involve the function

$$
g(x)=(1+x) \log (1+x)-x \text { for }-1<x<\infty .
$$

The function $g$ is strictly convex, strictly decreasing on $(-1,0)$, strictly increasing on $(0, \infty)$. It satisfies

$$
g(x)=\frac{1}{2} x^{2}+O\left(x^{3}\right) \quad \text { as } \quad x \longrightarrow 0 .
$$

More precisely,

$$
\frac{1}{2} x^{2} / 1+x<g(x)<\frac{1}{2} x^{2} \text { for } x>0,
$$


with the inequalities reversed for $x<0$. The function $g(x)$ is closely connected to a function which arises in the theory of large deviations theory. See, for example, pp. 100-101 of Kolchin, Sevastyanov, and Chistyakov [8].

We begin with a special case of Theorem (4b) in Freedman [4], restated here for ease of reference.

Lemma 3.6. Let $u>0$. Then

$$
P\{N>k p(1+u)\} \leqq \exp \{-g(u) k p\} .
$$

Combining (3.5) and (3.6) gives a version of Bernstein's inequality:

$$
P\{N>k p+m\} \leqq \exp \left\{-\frac{1}{2} m^{2} /(k p+m)\right\} \text { for } m>0 .
$$

Parenthetically, Theorem (4a) in Freedman [4] implies

$$
P\{N<k p-m\} \leqq \exp \left\{-\frac{1}{2} m^{2} / k p\right\} \quad \text { for } \quad m>0
$$

The next result is a variation on Lemma (7) of Freedman [5], and is proved the same way. (The condition $b>1 / \delta$ in that lemma is superfluous.)

LEMmA 3.9. Fix $\varepsilon>0$. There is a positive $\delta$ such that the ratio $P(N=a+b) / P(N=a)$ is bounded between $(1 \pm \varepsilon)$ times

$$
\left(\frac{a}{a+b}\right)^{1 / 2} \exp \left[-g\left(\frac{b}{a}\right) a\right]\left[\frac{(k-a) p}{a(1-p)}\right]^{b},
$$

for all integers $a, b$ and $k$ satisfying

$$
a>1 / \delta, \quad a+b>1 / \delta, \quad k>1 / \delta, \quad a+b<\delta k, \quad|b|<\delta \sqrt{k-a} .
$$

The next result gives a bound for binomial probabilities $P(N=\nu)$ in terms of the function $g$. Informally: for small values of $p$, large values of $k p$, and $\nu$ 's not too far from $k p, P(N=\nu)$ is asymptotic to

$$
\frac{1}{\sqrt{2 \pi k p}} \exp \{-g[(\nu-k p) / k p] k p\}
$$

This is a combination local central limit--large deviation result, holding uniformly in small $p$.

Lemma 3.10. Fix $\varepsilon>0$. There is a positive $\delta$ such that $P(N=\nu)$ is bounded between 


$$
(1 \pm \varepsilon) \frac{1}{\sqrt{2 \pi k p}} \exp \{-g[(\nu-k p) / k p] k p\}
$$

for all $k, p$ and $\nu$ satisfying

$$
p<\delta, \quad k p>1 / \delta, \quad|\nu-k p|<\delta k p, \quad|\nu-k p|<\delta \sqrt{k} .
$$

Proof. First, suppose $\nu$ is the integer part of $k p$, so $\nu=k p-\theta$ with $0 \leqq \theta<1$. Abbreviate $\bar{p}=1-p$, so $k-\nu=k \bar{p}+\theta$. By Stirling's formula, $P(N=\nu)$ is asymptotic to

$$
\frac{1}{\sqrt{2 \pi k}} \sqrt{\frac{k}{\nu} \frac{k}{k-\nu}}
$$

times

$$
\left(1-\frac{\theta}{k p}\right)^{-k p+\theta}\left(1+\frac{\theta}{k \bar{p}}\right)^{-k p-\varepsilon} .
$$

The first expression is asymptotic to $1 / \sqrt{2 \pi k p}$. The second is asymptotic to

$$
\left(1-\frac{\theta}{k p}\right)^{-k p}\left(1+\frac{\theta}{k \bar{p}}\right)^{-k p}
$$

By taking logs and expanding, the last is seen to be nearly 1 . Thus,

$$
P(N=[k p]) \approx 1 / \sqrt{2 \pi k p} .
$$

More explicitly, the ratio of the two sides of (3.11) converges to 1 as $\delta \rightarrow 0$, uniformly in $k$ and $p$ satisfying our conditions, namely, $p<\delta$ and $k p>1 / \delta$.

We now apply (3.9), with $a=[k p]$ and $b=\nu-a$ to see

$$
P(N=\nu) / P(N=[k p]) \approx \exp [-g(b / a) a] .
$$

First, as easily verified, the conditions of (3.9) hold. Second,

$$
\left(\frac{a}{a+b}\right)^{1 / 2} \approx 1
$$

indeed $a=k p+O(1)$ and $b=\nu-k p+O(1)$ is small relative to $k p$, by assumption. (The " $O(1)$ " terms are bounded as $\delta \rightarrow O$, uniformly in $k, p$ and $\nu$ satisfying our conditions.) Likewise,

$$
\left[\frac{(k-a) p}{a(1-p)}\right]^{b} \approx 1
$$

indeed, this expression is

$$
\left(1+\frac{\theta}{k \bar{p}}\right)^{b} /\left(1-\frac{\theta}{k p}\right)^{b},
$$


and $b=\nu-a=\nu-k p+O(1)$ is small relative to $k p$ and to $k \bar{p}$. This completes the proof of (3.12).

Recall that $a=[k p]$ and $b=\nu-a$. The final step in proving (3.10) is to show that

$$
\left|g\left(\frac{\nu-k p}{k p}\right) k p-g\left(\frac{b}{a}\right) a\right|
$$

is uniformly small. This tedious piece of calculus is omitted.

We now recall a bound on the tail probabilities of a normal random variable:

Suppose $U$ is a normal random variable with mean 0 and variance 1. Let $w>0$. Then $P\{U>w\}$ is bounded between

$$
\frac{1}{\sqrt{2 \pi}} \frac{1}{w+1 / w} \exp \left(-\frac{1}{2} w^{2}\right) \text { and } \frac{1}{\sqrt{2 \pi}} \frac{1}{w} \exp \left(-\frac{1}{2} w^{2}\right) \text {. }
$$

The following result is a generalization, as one sees by taking $f(x)=x^{2} / 2$.

Proposition 3.16. (a) Let $f$ be convex, and suppose $f(\infty)=\infty$. Then

$$
\int_{x}^{\infty} \exp [-f(u)] d u \leqq \frac{1}{f^{\prime}(x)} \exp [-f(x)]
$$

(b) Suppose $1 / f^{\prime}$ is convex. Then

$$
\int_{x}^{\infty} \exp [-f(u)] d u \geqq \frac{1}{f^{\prime}(x)+f^{\prime \prime}(x) / f^{\prime}(x)} \exp [-f(x)] .
$$

Proof. Claim (a). Let $F=\exp (-f)$. Then $F(\infty)=0$ and $F^{\prime}=-f^{\prime} F$. Now $f^{\prime}$ is monotone so

$$
f^{\prime}(x) \int_{x}^{\infty} F(u) d u \leqq \int_{x}^{\infty}-F^{\prime}(u) d u=F(x) .
$$

Claim (b). Let $\widetilde{F}=\left(1 / f^{\prime}\right) \exp (-f)$. Then $\widetilde{F}(\infty)=0$ and $\widetilde{F}^{\prime}=$ $-\left(1+f^{\prime \prime} / f^{\prime 2}\right) \exp (-f)$. So

$$
\left[1+\frac{f^{\prime \prime}(x)}{f^{\prime}(x)^{2}}\right] \int_{x}^{\infty} F(u) d u \geqq \int_{x}^{\infty}-\widetilde{F}^{\prime}(u) d u=\widetilde{F}(x) .
$$

Note. If $f$ is convex and $f^{\prime}$ is concave, then $1 / f^{\prime}$ is convex. This is the case for our function $g$. The concept of complete monotonicity is relevant here: see $\S$ XIII. 4 of Feller [3].

The next lemma extends Corollary (8) of Freedman [5]. It 
provides a bound for binomial tail probabilities, in terms of $g$, analogous to (3.15).

Proposition 3.17. Fix $\varepsilon>0$. There is a $\delta>0$ such that $P(N>k p+m)$ is bounded between

$$
(1 \pm \varepsilon) \frac{1}{\sqrt{2 \pi}} \frac{\sqrt{k p}}{m} \exp [-g(m / k p) k p \mid
$$

for all $k, p$ and $m$ satisfying

$$
p<\delta, k p>1 / \delta, \sqrt{k p} / \delta<m<\delta k p, m<\delta \sqrt{k} .
$$

Proof. We break the tail probability up into two parts:

$$
P\{N>k p+m\}=P_{1}+P_{2},
$$

where

$$
P_{1}=P\{k p+m<N \leqq k p+2 m\} \quad \text { and } \quad P_{2}=P\{N>k p+2 m\} .
$$

Later, we will show that $P_{2}$ is negligible, but that

$$
P_{1} \approx \frac{1}{\sqrt{2 \pi}} \frac{\sqrt{k p}}{m} \exp [-g(m / k p) k p]
$$

First, some preliminaries. To simplify the notation, let

$$
G(u)=\exp [-g(u / k p) k p] \text {. }
$$

Clearly,

$$
\frac{1}{\sqrt{2 \pi k p}} G(m) \text { is small relative to } \frac{1}{\sqrt{2 \pi}} \frac{\sqrt{k p}}{m} G(m),
$$

because $m$ is small relative to $k p$, by assumption. Likewise, in view of $(3.5)$

$$
G(2 m) \text { is small relative to } \frac{1}{\sqrt{2 \pi}} \frac{\sqrt{k p}}{m} G(m) \text {, }
$$

because $m$ is small relative to $k p$, but large relative to $\sqrt{k p}$. Next, we claim

$$
\sum_{i=m+1}^{\infty} G(i) \text { is bounded above by }\left(1+\varepsilon_{1}\right) \frac{k p}{m} G(m) .
$$

Indeed, $G$ is monotone decreasing, so

$$
\sum_{i=m+1} G(i)<\int_{m}^{\infty} G(u) d u<\frac{1}{g^{\prime}(m / k p)} G(m)
$$

by (3.16). But 


$$
g^{\prime}\left(\frac{m}{k p}\right)=\log \left(1+\frac{m}{k p}\right) \approx \frac{m}{k p}
$$

because $m$ is small relative to $k p$, by assumption. This proves (3.21).

We are now ready to prove part of (3.18), by estimating $P_{1}$ from above. Let $\nu$ range from $[k p]+m+1$ to $[k p]+2 m$, so $P_{1}=$ $\sum_{\nu} P(N=\nu)$. As (3.10) implies, $P_{1}$ is bounded above by

$$
\left(1+\varepsilon_{2}\right) \frac{1}{\sqrt{2 \pi} k p} \sum_{\nu} G(\nu-k p) .
$$

Because $G$ is decreasing, the expression (3.22) is bounded above by

$$
\frac{1}{\sqrt{2 \pi k p}} \sum_{i=m}^{2 m-1} G(i) \text {. }
$$

In view of (3.19) and (3.21),

$$
P_{1} \text { is bounded above by }
$$

$$
\left(1+\varepsilon_{3}\right) \frac{1}{\sqrt{2 \pi}} \frac{\sqrt{k p}}{m} \exp \{-g(m / k p) k p\} .
$$

We are now ready to prove the rest of (3.18), by estimating $P_{2}$ from below. Indeed, as (3.10) implies $P_{2}$ is bounded below by $\left(1-\varepsilon_{4}\right)$ times

$$
\frac{1}{\sqrt{2 \pi k p}} \sum_{\nu} G(\nu-k p) \geqq \frac{1}{\sqrt{2 \pi k p}} \sum_{i=m+1}^{2 m} G(i) .
$$

The right side of (3.24) is $T_{1}-T_{2}-T_{3}$, where:

$$
\begin{aligned}
T_{1} & =\frac{1}{\sqrt{2 \pi k p}} \sum_{i=m}^{\infty} G(i) \\
& \geqq \frac{1}{\sqrt{2 \pi k p}} \int_{m}^{\infty} G(u) d u \\
& \approx \frac{1}{\sqrt{2 \pi}} \frac{\sqrt{k p}}{m} G(m) \text { by }(3.16), \\
T_{2} & =\frac{1}{\sqrt{2 \pi k p}} G(m)=o\left[\frac{\sqrt{k p}}{m} G(m)\right] \text { by } \\
T_{3} & =\frac{1}{\sqrt{2 \pi k p}} \sum_{=2 m+1}^{\infty} G(i) \\
& \leqq\left(1+\varepsilon_{1}\right) \frac{1}{\sqrt{2 \pi}} \frac{\sqrt{k p}}{2 m} G(2 m) \text { by }(3.21) \\
& =O\left[\frac{V}{k p} G(m)\right] \text { by }(3.20),
\end{aligned}
$$


on recollecting that $\sqrt{k p / m}$ is small by assumption. This completes the proof of (3.18).

Our last job is to show that $P_{2}$ is negligible by comparison with $P_{1}$. But

$$
P_{2}=P(N>k p+2 m) \leqq G(2 m)
$$

by (3.6); now (3.18) and (3.20) can be used to complete the argument.

REMARK 3.25. Under the conditions of (3.17), $P(N<k p-m)$ can be bounded by the same expression. The argument is almost identical, because (3.10) is symmetric around $k p$. The only difference comes at the end:

$$
\begin{aligned}
P_{2}=P(N & <k p-2 m) \exp \left(-2 m^{2} / k p\right) \text { by }(3.8) \\
& <G(2 m) \text { by }(3.5) .
\end{aligned}
$$

Many of our arguments use the local central limit theorem. We state two versions for ease of reference. For the first version, assuming only a finite second moment, see page 517 of Feller [3]. For the second version, assuming a finite third moment, see page 197 of Petrov [10].

Suppose (1.1), and $E\left(X_{1}^{2}\right)<\infty$, and (1.6). Then uniformly in $j$,

$$
P\left(S_{n}=j\right)=\frac{1}{\sigma \sqrt{2 \pi n}} \exp \left\{\frac{1}{2}[(j-n \mu) / \sigma \sqrt{n}]^{2}\right\}+o(1 / \sqrt{n}) \text {. }
$$

Suppose (1.1), (1.2), and (1.6). Then uniformly in $j$

$$
P\left(S_{n}=j\right)=\frac{1}{\sigma \sqrt{2 \pi n}} \exp \left\{-\frac{1}{2}[(j-n \mu) / \sigma \sqrt{n}]^{2}\right\}+O(1 / n) .
$$

4. Proof of Theorem (1.8). The first step in the proof is to show that, with probability approaching one, the maximum does not occur in zones II, III, or IV as defined in (2.1). This part of the argument does not require third moments nor the full force of (1.5). Thus, for Lemmas (4.1), (4.3), and (4.5), we assume (1.1)-(1.6) with (1.2) replaced by $E\left(X_{1}^{2}\right)<\infty$ and (1.5) replaced by $n \rightarrow \infty$ and $k / \sqrt{n} \log n \rightarrow \infty$. We begin with zone IV.

LeMma 4.1. Let $y$ be any positive number. Let $M_{4}$ be the maximum of $N_{j}-k p_{j}$ over zone IV, namely the set of $j$ 's satisfying $|j-n \mu|>\sigma n^{3 / 4}$. Let $\theta_{n k}$ be the probability that $M_{4}>y(k \log n)^{1 / 2} \mid$ $n^{1 / 4}$. Then $\theta_{n k} \rightarrow 0$ as $n \rightarrow \infty$.

Proof. To begin with, $\theta_{n k}$ is bounded by the sum over $j$ in 
zone IV of

$$
P\left\{N_{j}-k p_{j}>y(k \log n)^{1 / 2} / n^{1 / 4}\right\}
$$

By Chebychev's inequality, the last displayed probability is at most

$$
y^{-2} n^{1 / 2} p_{j} / \log n
$$

Summing over $j$ in zone IV shows

$$
\theta_{n k} \leqq\left(y^{-2} n^{1 / 2} / \log n\right) \cdot P\left(\left|S_{n}-n \mu\right|>\sigma n^{3 / 4}\right)
$$

Using Chebyshev's inequality again,

$$
P\left(\left|S_{n}-n \mu\right|>\sigma n^{3 / 4}\right) \leqq n^{-1 / 2}
$$

So

$$
\theta_{n k} \leqq y^{-2} / \log n
$$

We turn now to zone III. It will be convenient to abbreviate

$$
m=\sqrt{\frac{k}{\sigma \sqrt{2 \pi n}} \log n} .
$$

This $m$ appears in (1.7) and is the leading term in (1.10); it will have some connection with the variable $m$ in $\S 3$.

LEMMA 4.3. Let $0<A<\infty$. Let $M_{A}$ be the maximum of $N_{j}$ $k p_{j}$ over $j$ in zone III, namely the set $S_{A}$ of $j$ with $A \sigma n^{1 / 2}<|j-n \mu| \leqq$ $\sigma n^{3 / 4}$. Fix any $\varepsilon$ with $0<\varepsilon<1$. Then there is an $A=A(\varepsilon)$ so large that

$$
P\left\{M_{A}>(1-\varepsilon) m\right\} \longrightarrow 0
$$

as $n$ and $k$ tend to infinity, satisfying the growth condition $k / V \bar{n} \log n \rightarrow \infty$.

Proof. Let $\theta$ satisfy $0<\theta<(1 / 4)(1-\varepsilon)^{2}$. Recall that $p_{j}=P\left(S_{n}=j\right)$. In view of the local central limit Theorem (3.26), there is an $A>0$ and a finite $n_{1}$ such that $p_{j}<\theta / \sigma \sqrt{2 \pi n}$ for all $j$ in $S_{A}$ provided $n>n_{1}$. From inequality (3.7),

$$
P\left\{N_{j}>k p_{j}+(1-\varepsilon) m\right\} \leqq \exp \left[-\frac{1}{2}(1-\varepsilon)^{2} \frac{m^{2}}{k p_{j}+(1-\varepsilon) m}\right] .
$$

We must now bound $m^{2} /\left[k p_{j}+(1-\varepsilon) m\right]$ from below. The choice of $A$ forces $k p_{j} \leqq k \theta / \sigma \sqrt{2 \pi n}$. The growth condition on $n$ and $k$ forces $(1-\varepsilon) m \leqq k \theta / \sigma \sqrt{2 \pi n}$ eventually. Then,

$$
\frac{m^{2}}{k p_{j}+(1-\varepsilon) m} \geqq \frac{m^{2}}{2 k \theta / \sigma \sqrt{2 \pi n}}=\frac{\log n}{2 \theta},
$$


and the right side of (4.4) is smaller than $1 / n$. Finally, $P\left\{M_{A}>\right.$ $(1-\varepsilon) m\}$ is bounded above by the sum over $j \in S_{A}$ of

$$
P\left\{N_{j}>k p_{j}+(1-\varepsilon) m\right\} .
$$

This sum comprises at most $2 \sigma n^{3 / 4}$ terms, each bounded by $1 / n$. This proves (4.3).

We turn now to zone II.

Lemma 4.5. Fix $A>\delta>0$. Let $M_{2}$ be the maximum of $N_{j}-$ $k p_{j}$ for $j$ in zone II, namely the set $S_{2}$ of $j$ with $\delta \sigma \sqrt{n} \leqq|j-n \mu| \leqq$ A $\sigma \sqrt{n}$. Let $m$ be defined by (4.2). Then, for $\varepsilon>0$ sufficiently small,

$$
P\left\{M_{2}>(1-\varepsilon) m\right\} \longrightarrow 0
$$

as $n$ and $k$ tend to infinity satisfying the growth condition $k / v^{\prime} \bar{n} \log n \rightarrow \infty$.

Proof. In view of the local central limit Theorem (3.26), there is a $\hat{o}_{1}>0$ and a finite $n_{1}$ such that for $n>n_{1}$,

$$
p_{j}<\left(1-\delta_{1}\right) / \sigma \sqrt{2 \pi n} \text { for all } j \text { satisfying }|j-n \mu|>\delta \sigma \sqrt{n} \text {. }
$$

Choose $\varepsilon$ positive with $(1-\varepsilon)^{2} /\left(1-\delta_{1}\right)>1$. Inequalities (3.7) and (4.6) imply, after some algebra:

$$
P\left\{N_{j}>k p_{j}+(1-\varepsilon) m\right\}<\exp \left[-\frac{1}{2} \beta \frac{\log n}{1+\theta_{n}}\right]
$$

where $\beta=(1-\varepsilon)^{2} /\left(1-\delta_{1}\right)>1$ by our choice of $\varepsilon$, and

$$
\theta_{n}=\frac{1-\varepsilon}{1-\delta_{1}} \sigma^{1 / 2}(2 \pi)^{1 / 4} n^{1 / 4}(\log n)^{1 / 2} / k^{1 / 2} \longrightarrow 0
$$

by the growth condition on $n$ and $k$.

Choose $\beta^{\prime}$ with $1<\beta^{\prime}<\beta$. For large $n$, the right side of (4.7) is at most $n^{-\beta^{\prime} / 2}$. Sum (4.7) over $j$ in $S_{2}$, comprising at most $2 A \sigma V \bar{n}$ terms, to see that

$$
P\left\{M_{2}>(1-\varepsilon) m\right\}<2 A \sigma \sqrt{n} n^{-\beta^{\prime} / 2} \longrightarrow 0 .
$$

We now proceed to zone I. Fix $\delta$ with $1<\delta<1 / 10$. For $-\infty \leqq a \leqq b \leqq \infty$, let $I_{a b}$ be the set of $j$ with $|j-n \mu|<\delta \sigma \sqrt{n}$ and

$$
a \sigma \sqrt{2 n / \log n} \leqq j-n \mu<b \sigma \sqrt{2 n \log n} .
$$


These intervals are all finite and nonrandom; $I_{-\infty \infty \infty}$ corresponds to all of zone I. Let

$$
W_{a b} \text { be the } \max \text { of } N_{j}-k p_{j} \text { for } j \text { in } I_{a b} \text {. }
$$

To state the main result concerning zone I, recall $z_{n}(x)$ from (1.10). As in (1.9), let $\Phi$ be the standard normal distribution function. We will now need finite third moments and the growth condition (1.5).

Proposition 4.9. Assume (1.1)-(1.6). Let $-\infty \leqq a \leqq b \leqq c \leqq$ $d \leqq \infty$. Then

$$
P\left\{W_{a b} \leqq z_{n}(x) \quad \text { and } \quad W_{c d} \leqq z_{n}(y)\right\}
$$

converges to $\exp \{-\sigma \sqrt{2} Q\}$, where

$$
Q=[\Phi(b)-\Phi(a)] e^{-x / 2}+[\Phi(d)-\Phi(c)\} e^{-y / 2} .
$$

The proof of (4.9) is a bit complicated. Here are some preliminary estimates.

LEMMA 4.10. The bounds given below apply uniformly to $j \in$ $I_{-\infty \infty}$, and the "o" errors are uniform in $j \in I_{-\infty \infty}$ as $n \rightarrow \infty$.

(a) $(j-n \mu)^{2} / \sigma^{2} n<\delta^{2}<1 / 100$.

(b) $p_{j}$ is bounded between

$$
\frac{1}{\sigma \sqrt{2 \pi n}}\left[1-\frac{1}{2}\left(1 \pm a_{\hat{\nu}}\right) \frac{(j-n \mu)^{2}}{\sigma^{2} n}\right]\left[1+o\left(\frac{1}{\log n}\right)\right]
$$

where $a_{\hat{o}}=(1 / 2) \delta^{2}$.

(c) $1 /\left[1-(1 / 2)\left(1 \pm a_{\hat{o}}\right)(j-n \mu)^{2} / \sigma^{2} n\right]$ is bounded between

$$
1+\frac{1}{2}\left(1 \pm b_{\delta}\right) \frac{(j-n \mu)^{2}}{\sigma^{2} n}
$$

where $b_{j}$ is a function of $\delta$ only and $b_{\delta}=O\left(\delta^{2}\right)$ as $\delta \rightarrow 0$.

Note. The notation in (b), although not standard, is convenient for our purposes and will be used throughout. To spell (b) out, there is a sequence $\varepsilon_{n}>0$ with $\varepsilon_{n} \log n \rightarrow 0$, such that for all $n$, and all $j$ in $I_{-\infty \infty}$, the probability $p_{j}$ is bounded above by

$$
\frac{1}{\sigma \sqrt{2 \pi n}}\left[1-\frac{1}{2}\left(1-a_{\tilde{o}}\right) \frac{(j-n \mu)^{2}}{\sigma^{2} n}\right]\left(1+\varepsilon_{n}\right)
$$

and below by

$$
\frac{1}{\sigma \sqrt{2 \pi n}}\left[1-\frac{1}{2}\left(1+a_{\hat{o}}\right) \frac{(j-n \mu)^{2}}{\sigma^{2} n}\right]\left(1-\varepsilon_{n}\right) .
$$


Proof. Part (a) is trivial, and then (b) follows from the local central limit Theorem (3.26), using the estimate

$$
1-x<e^{-x}<1-x+\frac{1}{2} x^{2} .
$$

Here, $x=(j-n \mu)^{2} / 2 \sigma^{2} n<(1 / 2) \delta^{2}$, so

$$
x-\frac{1}{2} x^{2}=\left(1-\frac{1}{2} x\right) x>\left(1-\frac{1}{4} \delta^{2}\right) x .
$$

Part (c) follows from the identity

$$
\frac{1}{1-x}=1+x+\frac{x^{2}}{1-x} \text {. }
$$

The probability in (4.9) will now be estimated from above and below. An upper bound derives from Mallows' inequality given in (3.1):

(4.11) The probability that $W_{a b} \leqq z_{n}(x)$ and $W_{c d} \leqq z_{n}(y)$ is at most

where

$$
\prod_{j \in I_{a b}} \lambda_{j}(x) \cdot \prod_{j \in I_{c d}} \lambda_{j}(y)
$$

$$
\lambda_{j}(x)=P\left\{N_{j} \leqq k p_{j}+z_{n}(x)\right\} .
$$

The rest of the upper-bounding is very similar to the lowerbounding, so details are omitted.

The lower bound will be derived from Lemma (3.2). In that lemma take

$$
\begin{aligned}
A_{j} & =\left\{N_{j} \leqq k p_{j}+z_{n}(x)\right\} \quad \text { for } \quad j \in I_{a b} \\
& =\left\{N_{j} \leqq k p_{j}+z_{n}(y)\right\} \quad \text { for } \quad j \in I_{c d} .
\end{aligned}
$$

To define $G_{j}$, let

(4.13a) $\quad K_{j}$ be the set of $i$ with $n \mu-\delta \sigma \sqrt{n}<i \leqq j$,

$$
\begin{gathered}
g_{j}=\sum_{i \in K_{j}} p_{i} \\
G_{j}=\left\{\sum_{i \in K_{j}} N_{i}>k g_{j}-M\right\},
\end{gathered}
$$

where

$$
M=(k \log n)^{1 / 2} .
$$

Since $I_{-\infty}$ includes at most $2 \delta \sigma n^{1 / 2}$ indices, (4.10b) shows

$$
g_{j}<0.8 \hat{o} \text { for all } j \in I_{-\infty \infty} \text {, for all large } n \text {. }
$$


Lemma (3.2) also involves $\sigma$-fields $\widetilde{F}_{j}$, defined as follows:

(4.15) $\widetilde{\mho}_{j}$ is the $\sigma$-field spanned by the $N_{\imath}$ as $i$ ranges over $K_{j}$. To apply Lemma (3.2), we must estimate $P\left(G_{j}\right)$.

Lemma 4.16. Define $G_{j}$ by (4.13). Then the sum of $1-P\left(G_{j}\right)$ over all $j \in I_{-\infty}$ tends to 0 as $n$ and $k$ tend to infinity satisfying (1.5).

Proof. Clearly, $\sum_{i \in \kappa_{j}} N_{i}$ is binomial: the number of trials is $k$, and the success probability is $g_{j}$. From (3.8),

$$
1-P\left(G_{j}\right) \leqq \exp \left[-\frac{1}{2} M^{2} / k g_{j}\right] .
$$

Replace $g_{j}$ by the upper bound (4.14): eventually,

$$
1-P\left(G_{j}\right) \leqq \exp \left(-\frac{1}{2} M^{2} / k \hat{\delta}\right)=n^{-1 / 2 \tilde{o}}<n^{-5}
$$

for all $j \in I_{-\infty \infty \infty}$. But $I_{--\infty \infty \infty}$ only comprises $O\left(n^{1 / 2}\right)$ terms.

We are now ready to establish the basic lower bound.

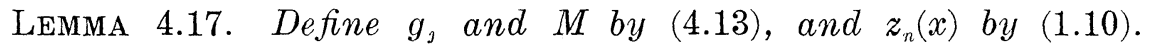
Let $N_{j}^{\prime}$ be binomial, with success probability $p_{j}^{\prime}=p_{j} /\left(1-g_{j-1}\right)$ and the number of trials $k_{j}^{\prime}$, the integer part of $k\left(1-g_{j-1}\right)+M$. Let

$$
\lambda_{j}(x)=P\left\{N_{j}^{\prime} \leqq k p_{j}+z_{n}(x)\right\} .
$$

Then $P\left\{W_{a b} \leqq z_{n}(x)\right.$ and $\left.W_{c d} \leqq z_{n}(y)\right\}$ is bounded below by

$$
\prod_{j \in I_{a b}} \lambda_{j}(x) \cdot \prod_{j \in J_{c d}} \lambda_{j}(y)-\sum_{j \in I-\infty \infty \infty}\left[1-P\left(G_{j}\right)\right] .
$$

Proof. Given $\widetilde{\mho}_{j-1}$, as defined in (4.15), the conditional distribution of $N_{j}$ is still binomial; the conditional success probability is $p_{j}^{\prime}$, as defined above; the number of trials $T_{j}$ is an $\widetilde{\mho}_{j-1}$-measurable random variable:

On $G_{j-1}$, however, $T_{j} \leqq k_{j}^{\prime}$. So

$$
T_{j}=k-\sum_{i \in K_{j-1}} N_{\imath} .
$$

$$
P\left\{N_{j} \leqq k p_{j}+z_{n}(x) \mid \mathfrak{F}_{j-1}\right\} \geqq \lambda_{j}(x) \text { on } G_{j-1}:
$$

heuristically, the more you toss the coin, the more heads you get. Lemma (3.2) completes the proof.

We must now estimate $\lambda_{\jmath}$. Here are some preliminaries. 
Lemma 4.18. Define $\lambda_{j}$ as in (4.17). So,

$$
\lambda_{j}(x)=P\left\{N_{j}^{\prime} \leqq k_{j}^{\prime} p_{j}^{\prime}+h\right\}
$$

where

$$
h=z_{n}(x)+k p_{j}-k_{j}^{\prime} p_{j}^{\prime} .
$$

The bounds and error terms given below are uniform over $j \in I_{-\infty \infty}$; the " $O$ " and " $O$ " are as $k$ and $n$ approach infinity, satisfying condition (1.5) that $k / \sqrt{n}(\log n)^{3} \rightarrow \infty$.

(a) $k_{j}^{\prime} p_{j}^{\prime}$ is bounded between

$$
\frac{k}{\sigma \sqrt{2 \pi n}}\left[1-\frac{1}{2}\left(1 \pm a_{\bar{\delta}}\right) \frac{(j-n \mu)^{2}}{\sigma^{2} n}\right]\left[1+o\left(\frac{1}{\log n}\right)\right]
$$

with $a_{\hat{\delta}}$ of $(4.10 \mathrm{~b})$.

(b) $h=z_{n}(x)\left[1+O\left(n^{-1 / 4}\right)\right]$

(c) $h^{3}=o\left(k_{j}^{\prime} p_{j}^{\prime}\right)^{2}$

(d) $h^{2} / k_{j}^{\prime} p_{j}^{\prime}$ is bounded between

$$
\log n-2 \log \log n+x+\frac{1}{2}\left(1 \pm 2 b_{\delta}\right) \frac{(j-n \mu)^{2}}{\sigma^{2} n / \log n}+o(1)
$$

with the $b_{\delta}$ of $(4.10 \mathrm{c})$.

(e) Recall the definition of $g(x)$ at (3.3).

Then $g\left(h / k_{j}^{\prime} p_{i}^{\prime}\right) k_{j}^{\prime} p_{j}^{\prime}$ is bounded between

$$
\frac{1}{2} \log n-\log \log n+\frac{1}{2} x+\frac{1}{4}\left(1 \pm 2 b_{\delta}\right) \frac{(j-n \mu)^{2}}{\sigma^{2} n / \log n}+o(1) .
$$

Proof. Part (a). From the definitions of $p_{j}^{\prime}$ and $k_{j}^{\prime}$ given in (4.17),

$$
k_{j}^{\prime} p_{j}^{\prime}=k p_{j}\left[1+\frac{M-\theta}{k\left(1-g_{j-1}\right)}\right],
$$

where $0 \leqq \theta \leqq 1$. By (4.13d) and condition (1.5), $(M-\theta) / k=o(1 /$ $\log n)$; and $g_{j}<\delta$ eventually, by (4.14). Then (4.10b) completes the argument for part (a).

Part (b). Recall $h$ as defined in the statement of the lemma. Continuing from (4.19),

$$
\frac{h}{z_{n}(x)}-1=-\frac{(M-\theta)}{z_{n}(x)} \frac{p_{j}}{1-g_{j-1}}=O\left(n^{-1 / 4}\right)
$$

as in (a), using the Definition (1.10) of $z_{n}(x)$.

Part (c). This is immediate from (a), (b), and condition (1.5). This is the first time that the full force of (1.5) has been used. tween

Part (d). Using (a), (b), and (4.10c), $h^{2} / k_{i}^{\prime} p_{j}^{\prime}$ is bounded be- 
$(\log n-2 \log \log n+x)\left[1+\frac{1}{2}\left(1 \pm b_{\delta}\right) \frac{(j-n \mu)^{2}}{\sigma^{2} n}\right]\left[1+o\left(\frac{1}{\log n}\right)\right]$.

Of course, $(2 \log \log n-x) / \log n<b_{\delta}$ eventually, proving (d).

Part (e). This follows from (c) and (d), by using inequality (3.4).

The proof of Proposition (4.9). We pick up the argument from (4.17). The problem is to put a lower bound on $\prod_{j \in I_{a b}} \lambda_{j}(x)$, the other factor being similar. Defining $h$ as in (4.18),

$$
\begin{aligned}
1-\lambda_{j}(x) & =P\left\{N_{j}^{\prime}>n p_{j}+z_{n}(x)\right\} \\
& =P\left\{N_{j}^{\prime}>k_{j}^{\prime} p_{j}^{\prime}+h\right\} .
\end{aligned}
$$

Fix an $\varepsilon>0$. Then Proposition (3.17) implies that eventually, for all $j \in I_{-\infty \infty}, 1-\lambda_{j}(x)$ is bounded between

$$
(1 \pm \varepsilon)\left(k_{j}^{\prime} p_{j}^{\prime} / 2 \pi\right)^{1 / 2} \frac{1}{h} \exp \left[-g\left(h / k_{j}^{\prime} p_{j}^{\prime}\right) k_{j}^{\prime} p_{j}^{\prime}\right] .
$$

"Eventually" means for all $k$ and $n$, with $n$ and $k / \sqrt{n} \log n$ sufficiently large.

We pause to verify the conditions of the lemma being appealed to: $p_{j}^{\prime}=p_{j} /\left(1-g_{j-1}\right)$ is small by $(4.10 \mathrm{~b})$ and $(4.14) ; k_{j}^{\prime} p_{j}^{\prime}$ is large by (4.18d); $h^{2} / k_{j}^{\prime} p_{j}^{\prime}$ is large by (4.18d); $h / k_{j}^{\prime} p_{j}^{\prime}$ is small by (4.18c); and $h / k^{1 / 2}$ is small by (4.18b). Thus, the bound (4.20) is established.

The exponent in (4.20) was estimated in (4.18e); the factor $k_{j}^{\prime} p_{j}^{\prime}$ in (4.18a); and the factor $h$ in (4.18b). We conclude that eventually, for all $j$ in $I_{-\infty \infty}, 1-\lambda_{j}(x)$ is bounded between

$$
(1 \pm \varepsilon)\left(1 \pm c_{\delta}\right) \sigma \sqrt{2} e^{-x / 2}
$$

times

$$
\frac{1}{\sqrt{2 \pi}} \frac{1}{\sqrt{2 \sigma^{2} n / \log n}} \exp \left\{-\frac{1}{4}\left(1 \pm 2 b_{\delta}\right) \frac{(j-n \mu)^{2}}{\sigma^{2} n / \log n}\right\}
$$

where $c_{\delta}$ is a function of $\delta$ only and $c_{\delta}=O\left(\delta^{2}\right)$ as $\delta \rightarrow 0$.

Arguing as in Proposition (2.2), we get a lower bound for the probability in (4.9), of the form $(1-3 \varepsilon)\left(1-d_{\delta}\right) \exp \left(-Q_{\delta}\right)$, where

$$
\begin{aligned}
Q_{\delta} & =\sigma \sqrt{2} e^{-x / 2}\left[\Phi\left(f_{\delta} b\right)-\Phi\left(f_{\delta} a\right)\right] \\
& +\sigma \sqrt{2} e^{-y / 2}\left[\Phi\left(f_{\delta} d\right)-\Phi\left(f_{\hat{\delta}} c\right)\right]
\end{aligned}
$$

with $d_{\delta}=O\left(\delta^{2}\right)$ and $f_{\delta}=1+O\left(\delta^{2}\right)$. It is at this point that the uniformity in $j$ was critical.

An upper bound of similar form can be obtained by essentially the same argument, starting from (4.11) instead of (4.17). Then the proof can be completed as in (2.2). 
Our last main job in proving (1.8) is showing that $\max \left(N_{j}-k p_{j}\right)$ is assumed at a unique index $j$, with probability approaching one as $n$ and $k / \sqrt{n}(\log n)^{3}$ tend to $\infty$. The following heuristic discussion may make the argument easier to follow. Inspection of (1.8) suggests that the maximum is likely to occur only for $j$ 's within $O(n / \log n)^{1 / 2}$ of $n \mu$. Call these the critical $j$ 's.

There are $O(n / \log n)^{1 / 2}$ critical $j$ 's .

Recall $m$ from (4.2). The range of likely values for the maximum is from

$$
m\left(1-\frac{2 \log \log n}{\log n}-\frac{b}{\log n}\right)^{1 / 2} \doteq m\left(1-\frac{2 \log \log n}{2 \log n}-\frac{b}{2 \log n}\right)
$$

to

$$
m\left(1-\frac{2 \log \log n}{\log n}+\frac{b}{\log n}\right)^{1 / 2} \doteq m\left(1-\frac{2 \log \log n}{2 \log n}+\frac{b}{2 \log n}\right) .
$$

Call these the critical values. In what follows we write $a_{n} \sim b_{n}$ if $\lim a_{n} / b_{n}>0$ and $\overline{\lim } a_{n} / b_{n}<\infty$.

$$
\begin{aligned}
& \text { There are the order of } m / \log n \sim k^{1 / 2} n^{-1 / 4}(\log n)^{-1 / 2} \text { critical } \\
& \text { values for the maximum. }
\end{aligned}
$$

Each of these critical values for the maximum corresponds to some value $i$ for an $N_{j}$ of around

$$
\begin{aligned}
k p_{j} & +m\left(1-\frac{2 \log \log n}{\log n}\right)^{1 / 2} \\
& \doteq k p_{j}+\left(k p_{j}\right)^{1 / 2}(\log n-2 \log \log n)^{1 / 2} .
\end{aligned}
$$

Now $N_{j}-k p_{j}$ is essentially normal with mean 0 and variance $k p_{j} \sim$ $k / \sqrt{n}$, so

$$
\begin{aligned}
P\left(N_{j}\right. & =i) \doteq \frac{1}{\sqrt{2 \pi k p_{j}}} \exp \left[-\frac{1}{2} \frac{\left(i-k p_{j}\right)^{2}}{k p_{j}}\right] \\
& \sim k^{-1 / 2} n^{1 / 4} \exp \left[-\frac{1}{2} \log n+\log \log n\right] \\
& =k^{-1 / 2} n^{-1 / 4} \log n .
\end{aligned}
$$

Furthermore, the $N_{j}$ are nearly independent. The chance that the maximum occurs at two different indices is bounded by the sum, over the critical $j, j^{\prime}, i$ and $i^{\prime}$ satisfying

$$
i-k p_{j}=i^{\prime}-k p_{j^{\prime}}
$$

of 


$$
P\left(N_{j}=i \quad \text { and } \quad N_{j^{\prime}}=i^{\prime}\right) \sim k^{-1} n^{-1 / 2}(\log n)^{2} .
$$

The number of critical $i$ 's was estimated in (4.22): to each, there corresponds at most one $i^{\prime}$ by (4.23), "at most" because $i^{\prime}$ has to be an integer. The number of pairs $j, j^{\prime}$ is $O(n / \log n)$ by (4.21). So the chance that the maximum occurs at two different indices is of order

$$
\begin{aligned}
& n(\log n)^{-1} \cdot k^{1 / 2} n^{-1 / 4}(\log n)^{-1 / 2} \cdot k^{-1} n^{-1 / 2}(\log n)^{2} \\
& \quad=k^{-1 / 2} n^{1 / 4}(\log n)^{1 / 2}
\end{aligned}
$$

This last quantity tends to zero because of the growth condition (1.5).

Returning to rigorous argument, recall $z_{n}(x)$ defined in (1.10) and $I_{a b}$ defined by (4.8). The main estimate is the following.

Lemma 4.24. Assume (1.1)-(1.6). Fix positive finite numbers a and $b$. Then, uniformly over pairs of indices $j \neq j^{\prime}$ in $I_{-a a}$, and pairs of integers $i, i^{\prime}$ satisfying

$$
z_{n}(-b) \leqq i-k p_{j}=i^{\prime}-k p_{j^{\prime}} \leqq z_{n}(b)
$$

we have

$$
P\left(N_{j}=i\right)=O\left(k^{-1 / 2} n^{-1 / 4} \log n\right)
$$

and

$$
P\left(N_{j^{\prime}}=i^{\prime} \mid N_{j}=i\right)=O\left(k^{-1 / 2} n^{-1 / 4} \log n\right) .
$$

Proof. The first assertion (4.26) follows from Lemma (3.10), the requisite estimates for $p_{j}$ being given by (4.10). To make this valid, the conditions of (3.10) must be verified. Then, one argues from (4.25) and (1.5) that $\left(i-k p_{j}\right)^{3}=o\left(k p_{j}\right)^{2}$, so

$$
g\left[\left(i-k p_{j}\right) / k p_{j}\right] k p_{j}=\frac{1}{2}\left(i-k p_{j}\right)^{2} / k p_{j}+o(1) .
$$

But, using (4.22) again,

$$
\left(i-k p_{j}\right)^{2}=\frac{k}{\sigma \sqrt{2 \pi n}}[\log n-2 \log \log n+O(1)] .
$$

And by (4.10),

$$
k p_{j}=\frac{k}{\sigma \sqrt{2 \pi n}}\left[1+O\left(\frac{1}{\log n}\right)\right] .
$$

So

$$
g\left[\left(i-k p_{j}\right) / k p_{j}\right] k p_{j}=\frac{1}{2} \log n-\log \log n+O(1)
$$


We omit the other details in the proof of (4.26).

For the second assertion (4.27), given $N_{j}=i$, the conditional distribution of $N_{j^{\prime}}$ is binomial with success probability $\hat{p}=p_{j^{\prime}} /$ $\left(1-p_{j}\right)$ and number of trials $\hat{k}=k-i$. Some preliminary estimates are needed before appealing to Lemma (3.10). All " $O$ " and " $o$ " estimates are uniform over $j \neq j^{\prime}$ in $I_{-a a}$ and $i, i^{\prime}$ satisfying (4.25), as $n$ and $k / \sqrt{n}(\log n)^{3}$ tend to infinity. We will show that

$$
\begin{gathered}
\hat{k} \hat{p}=k p_{j^{\prime}}[1+o(1 / \log n)] \\
\left(i^{\prime}-\hat{k} \hat{p}\right)^{2} / \hat{k} \hat{p}=\log n-2 \log \log n+O(1) .
\end{gathered}
$$

Assume these bounds for the moment. Another application of Lemma (3.10) shows that $P\left(N_{j^{\prime}}=i^{\prime} \mid N_{j}=i\right)$ is of order

$$
(\hat{k} \hat{p})^{-1 / 2} \exp \left[-\frac{1}{2}\left(i^{\prime}-\hat{k} \hat{p}\right)^{2} / \hat{k} \hat{p}\right] \text {. }
$$

Now use (4.28) and (4.29) as before to complete the proof.

We now prove (4.28) and (4.29). Let

$$
\theta=\hat{k} \hat{p}-k p_{j^{\prime}}=\left(k p_{j}-i\right) p_{j^{\prime}} /\left(1-p_{j}\right) .
$$

Abbreviate $h=i^{\prime}-k p_{j^{\prime}}=i-k p_{j}$. Then (b) of Lemma (4.10) implies $\theta / h=-p_{j^{\prime}} /\left(1-p_{j}\right)=O(1 / \sqrt{n})$. Condition (4.25) makes $h$ of order $k^{1 / 2} n^{-1 / 2}(\log n)^{1 / 2}$, and then $\theta=O\left(k^{1 / 2} n^{-3 / 4}(\log n)^{1 / 2}\right)$. Finally $k p_{j^{\prime}}$ is of order $k / \sqrt{n}$, again by (b) of Lemma (4.10). To summarize:

$$
\theta=O\left[k^{1 / 2} n^{-3 / 4}(\log n)^{1 / 2}\right], h \approx k^{1 / 2} n^{-1 / 4}(\log n)^{1 / 2}, k p_{j^{\prime}} \approx k n^{-1 / 2} .
$$

Now we can prove (4.28):

$$
\begin{aligned}
\hat{k} \hat{p} & =k p_{j^{\prime}}+\theta \\
& =k p_{j^{\prime}}+O\left[k^{1 / 2} n^{-3 / 4}(\log n)^{1 / 2}\right] \\
& =k p_{j^{\prime}}\left\{1+O\left[k^{-1 / 2} n^{-1 / 4}(\log n)^{1 / 2}\right]\right\} \\
& =k p_{j^{\prime}}[1+o(1 / \log n)] .
\end{aligned}
$$

To prove (4.29), note that $i^{\prime}-\hat{k} \hat{p}=h-\theta$. Now

$$
\left(i^{\prime}-\hat{k} \hat{p}\right)^{2} / \hat{k} \hat{p}=\left(h^{2}-2 \theta h+\theta^{2}\right) / \hat{k} \hat{p}=h^{2} / \hat{k} \hat{p}+o(1) .
$$

From (4.25)

$$
h^{2}=\frac{k}{\sigma \sqrt{2 \pi n}}[\log n-2 \log \log n+O(1)] .
$$

In view of (4.10),

$$
\hat{k} \hat{p}=\frac{k}{\sigma \sqrt{2 \pi n}}[1+O(1 / \log n)] .
$$




$$
h^{2} / \hat{k} \hat{p}=\log n-2 \log \log n+O(1) .
$$

We can now prove the uniqueness assertion in (1.8).

Proposition 4.31. Assume (1.1-1-6). Then $\max _{j}\left(N_{j}-k p_{j}\right)$ is assumed at a unique index $j$, with probability approaching one.

Proof. We first establish:

Given $\varepsilon>0$, there are large, finite numbers $a$ and $b$, such that eventually, except for probability $\varepsilon, \max _{j}\left(N_{j}-k p_{j}\right)$ is assumed only for indices in $I_{-a a}$, and is between $z_{n}(-b)$ and $z_{n}(b)$.

"Eventually" means for sufficiently large values of $n$ and $k / n^{1 / 2}(\log n)^{3}$. Indeed, for any $b$, except for probability less than $\varepsilon / 5$, the maximum over zone IV is eventually smaller than $z_{n}(-b)$ by (4.1). Likewise for zone III by (4.3), and zone II by (4.5). As a matter of notation, zone $I$ is $I_{-\infty \infty}$ and can be dealt with by (4.9). There are $a$ and $b$ so large that eventually, except for probability $\varepsilon / 5$,

$$
z_{n}(-b)<\max \left\{N_{j}-k p_{j} \text { for } j \text { in } I_{-\infty \infty}\right\}<z_{n}(b) .
$$

Finally, choose $a$ so large that eventually, except for probability $\varepsilon / 5, N_{j}-k p_{j}<z_{n}(-b)$ for all $j \in I_{-\infty \infty} \mid I_{-a a}$. This completes the proof of (4.32).

The next two estimates are easily checked.

(4.33) The number of pairs $\left(j, j^{\prime}\right)$ with $j^{\prime} \neq j$ in $I_{-a a}$ is $O(n / \log n)$.

The number of integers $i$ with $z_{n}(-b) \leqq i-k p_{j}<z_{n}(b)$ is at most

$$
z_{n}(b)-z_{n}(-b)=O\left[k^{1 / 2} n^{-1 / 4}(\log n)^{-1 / 2}\right] .
$$

Now, Lemma (4.24) and relations (4.33-34) enable us to estimate the chance of finding two distinct indices $j^{\prime} \neq j$ in $I_{-a a}$ with $N_{j}-k p_{j}=$ $N_{j^{\prime}}-k p_{j^{\prime}}$ in the critical range from $z_{n}(-b)$ to $z_{n}(b)$. Take the number of pairs $j \neq j^{\prime}$ in $I_{-a a}$, and multiply by the number of integers $i$ with $i-k p_{j}$ in the critical range. Each $i$ is a possible value for $N_{j}$, and associated with it is at most one possible value $i^{\prime}$ for $N_{j^{\prime}}$ satisfying $i-k p_{j}=i^{\prime}-k p_{j^{\prime}}$. Then, we multiply by $P\left(N_{j}=i\right) \cdot P\left(N_{j^{\prime}}=i^{\prime} \mid N_{j}=i\right)$. The result is $O\left[k^{-1 / 2} n^{-1 / 4}(\log n)^{1 / 2}\right] \rightarrow 0$.

The proof of Theorem (1.8) can now be given.

Proof of Theorem (1.8). As Proposition (4.31) implies, $M_{n k}$ is assumed at a unique location $L_{n k}$, with probability approaching one. 
Lemmas (4.1), (4.3), and (4.5) imply that with probability approaching one, $L_{n k}$ is in zone I. Now, Proposition (4.9) implies (1.8) by the same argument which showed that equation (2.4) implies Proposition (2.2).

5. Moment assumptions. We have been assuming (1.2) that $E\left|X_{1}^{3}\right|<\infty$. An argument sketched later in this section will prove Theorem (1.8) under the weaker hypothesis

$$
E\left\{\left|X_{1}\right|^{2}\left[\log \left(1+\left|X_{1}\right|\right)\right]^{2+\delta}\right\}<\infty \text { for some } \delta>0 \text {. }
$$

However, if only

$$
E\left\{\left|X_{1}\right|^{2}\left[\log \left(1+\left|X_{1}\right|\right)\right]^{\delta}\right\}<\infty \text { for some } \delta \text { with } 0<\delta<1,
$$

the conclusions of the main Theorem (1.8) become false. This is demonstrated by Theorem (5.4) below, which gives the correct asymptotic formulae for a particular random variable satisfying (5.2) but not (5.1). The present discussion leaves unresolved the case $E\left\{\left|X_{1}\right|^{2}\left[\log \left(1+\left|X_{1}\right|\right)\right]^{1+\delta}\right\}<\infty$ for some $\delta$ with $0 \leqq \delta \leqq 1$.

Theorem (5.4) uses an Edgeworth-like correction in the local central limit theorem for a random variable without third moments, given in (5.23). The techniques used are similar to those in Pitman [11]. Some related work can be found in Cramer [1] or Takeuchi and Akahira [12, 13].

We now define a class of probability distributions on the integers. Let $a>1$ be given. Let $q=q_{a}$ be the symmetric probability on the integers with

$$
\left\{\begin{array}{l}
q(0)=q(-1)=q(1)=0 \\
q(j)=b /\left[j^{3}(\log |j|)^{a}\right] \text { for } j= \pm 2, \pm 3, \cdots, \\
b \text { is chosen so that } \sum_{j} q(j)=1
\end{array}\right.
$$

As for Theorem (1.8), let $\sigma^{2}$ be the variance of $q$. Let $X_{1}, X_{2}, \cdots$ be independent with common distribution (5.3), and write $S_{n}=X_{1}+$ $\cdots+X_{n}$. Take $k$ independent copies of $S_{n}$. Let $N_{j}$ be the number of these sums which are equal to $j$. Let $M_{n k}=\max _{j}\left[N_{j}-k P\left(S_{n}=j\right)\right]$.

THEOREM 5.4. Let $X_{1}, X_{2}, \cdots$ be independent, having common distribution (5.3), with $1.5<a \leqq 2$. Let $c=b 2^{a-1} /(a-1)$. Define

$$
z_{n}(x, a)=\sqrt{\frac{k}{\sigma \sqrt{2 \pi n}}} \sqrt{\log n+c(\log n)^{2-a}-2 \log \log n+x} .
$$

Then, with probability approaching one, $M_{n k}$ is taken on at a unique location $L_{n k}$, and the probability that 


$$
L_{n k}<y \sigma \sqrt{2 n / \log n} \text { and } M_{n k}<z_{n}(x, a)
$$

converges to

$$
\Phi(y) \exp \left[-\sigma \sqrt{2} e^{-x / 2}\right]
$$

as $n$ and $k$ tend to infinity satisfying the growth condition (1.5).

Note that $q$ in (5.3) has a second moment, but just barely. In particular

$$
\sum j^{2}[\log (1+|j|)]^{\delta} q(j)
$$

is finite provided $\delta<a-1$, but infinite for $\delta \geqq a-1$. Note too that $z_{n}(x, a)$ defined in (5.5) is different from $z_{n}(x)$ of (1.10). When $a=2$, the difference is not so dramatic - a constant under the square root sign. But when $a<2$, there is an extra term going to infinity, namely $c(\log n)^{2-a}$.

To prove (5.4), sharp estimates of $P\left(S_{n}=j\right)$ are needed. This is done by approximating the characteristic function of $X_{1}$. We begin with some preliminary estimates. The first lemma is implicit in Pitman [11].

Lemma 5.6. Suppose the real numbers $u_{n}$ are nonnegative, nonincreasing and (weakly) convex:

$$
u_{n} \geqq 0, u_{n} \geqq u_{n+1}, u_{n}-u_{n+1} \geqq u_{n+1}-u_{n+2} .
$$

Suppose too that $u_{n} \rightarrow 0$ as $n \rightarrow \infty$. Let $S$ be the (conditionally convergent) sum $u_{0}-u_{1}+u_{2}-u_{3}+\cdots$. Then

$$
\frac{1}{2} u_{0} \leqq S \leqq u_{0}-\frac{1}{2} u_{1}
$$

Proof. Clearly,

$$
\begin{aligned}
S & =\left(u_{0}-u_{1}\right)+\left(u_{2}-u_{3}\right)+\cdots \\
& \geqq\left(u_{1}-u_{2}\right)+\left(u_{3}-u_{4}\right)+\cdots \text { by convexity } \\
& =u_{0}-S .
\end{aligned}
$$

This proves the first inequality. For the second,

$$
S=u_{0}-\left(u_{1}-u_{2}+u_{3}-\cdots\right) \leqq u_{0}-\frac{1}{2} u_{1},
$$

because $u_{1}-u_{2}+u_{3}-\cdots \geqq(1 / 2) u_{1}$ by the first inequality applied to the sequence $u_{1}, u_{2}, \cdots$.

The next lemma is at the heart of the approximations in this section. It is abstracted from Theorem 2 of Pitman [11]. We work 
with conditionally convergent Riemann integrals. Such integrals are denoted by using an arrow over the integral sign.

LEMMA 5.7. Let $H(x)$ be a convex function on $[0, \infty)$ which decreases to 0 . Then, for any $t>0$,

$$
\frac{1}{2} I_{0} \leqq t \int_{0}^{\rightarrow \infty} H(x) \sin (t x) d x \leqq I_{0}-\frac{1}{2} I_{1}
$$

where

$$
I_{0}=\int_{0}^{\pi} H(x / t) \sin x d x \quad \text { and } \quad I_{1}=\int_{0}^{\pi} H[(x+\pi) / t] \sin x d x .
$$

Proof. By changing variables, it is enough to do the case $t=1$. Abbreviate $h_{j}(x)=H(x+j \pi)$. Split at multiples of $\pi$ to see that the integral to be estimated equals the conditionally convergent sum

$$
\sum_{j=0}^{\infty} \int_{j \pi}^{(j+1) \pi} H(x) \sin x d x=\sum_{j=0}^{\infty} \int_{0}^{\pi}(-1)^{j} h_{j}(x) \sin x d x .
$$

Group the terms as $\left(u_{0}-u_{1}\right)+\left(u_{2}-u_{3}\right)+\cdots$ to get

$$
\sum_{k=0}^{\infty} \int_{0}^{\pi}\left[h_{2 k}(x)-h_{2 k+1}(x)\right] \sin x d x .
$$

Because $H$ is monotone decreasing, $h_{2 k} \geqq h_{2 k+1}$ : so the last sum is absolutely convergent, and we can take $\sum$ inside the integral by Fubihi's theorem. As a result,

$$
\int_{0}^{\rightarrow \infty} H(x) \sin x d x=\int_{0}^{\pi} \sum_{k=0}^{\infty}\left[h_{2 k}(x)-h_{2 k+1}(x)\right] \sin x d x .
$$

Now $H$ is convex, so for each $x$ the sequence $h_{0}(x), h_{1}(x), \cdots$ is convex. By (5.6),

$$
\frac{1}{2} h_{0}(x) \leqq \sum_{k=0}^{\infty}\left[h_{2 k}(x)-h_{2 k+1}(x)\right] \leqq h_{0}(x)-\frac{1}{2} h_{1}(x) \text {. }
$$

This completes the proof.

We will now apply this result to $H$ 's of order $1 /(\log x)^{\alpha}$, at infinity, and evaluate the corresponding integrals $I_{0}$ and $I_{1}$ in (5.7). A calculus estimate will be needed.

Lemma 5.11. Let $\alpha$ be a positive number and $x$ a real number. Then,

(a) $1 /(1+x)^{\alpha}>1-\alpha x$ for $x>-1$.

(b) $1 /(1+x)^{\alpha}<1-\alpha x+2^{\alpha+1} \alpha(\alpha+1) x^{2}$ for $x \geqq-1 / 2$. 
(c) $1 /(1-x)^{\alpha}<1+k x$ for $0 \leqq x \leqq 1 / 2$, with $k$ finite but depending on $\alpha$.

Proof. Expand $1 /(1+x)^{\alpha}$ in a Taylor series with remainder.

Lemma 5.12. Let $\alpha$ be a positive number. Then, as $t \rightarrow 0^{+}$,

$$
\int_{\sqrt{t}}^{\pi}\left(\log \frac{x}{t}\right)^{-\alpha} \sin x d x
$$

and

$$
\int_{\sqrt{t}}^{\pi}\left(\log \frac{x+\pi}{t}\right)^{-\alpha} \sin x d x
$$

are both equal to

$$
2\left(\log \frac{1}{t}\right)^{-\alpha}+O\left(\log \frac{1}{t}\right)^{-\alpha-1}
$$

Proof. We will only do the first integral, the second being similar. Write the first integrand as

$$
\left(\log \frac{1}{t}\right)^{-\alpha}\left(1+\frac{\log x}{\log 1 / t}\right)^{-\alpha} \sin x .
$$

Now $x \geqq \sqrt{t}$, so $(\log x) /(\log 1 / t) \geqq-1 / 2$, and $(5.11 \mathrm{a}-\mathrm{b})$ can be used to estimate the middle factor in the display. The integrals which result are evaluated as follows:

$$
\begin{aligned}
& \int_{\sqrt{t}}^{\pi} \sin x d x=2+O(t) \\
& \int_{0}^{\pi} \sin x|\log x|^{j} d x<\infty \text { for } j=1 \text { or } 2 .
\end{aligned}
$$

The following calculus facts are needed to estimate the tails of the distribution (5.3).

LEMMA 5.13. Let $\alpha>0$ and $\beta>1$.

(a) Define

$$
f(\alpha, \beta, u)=\int_{u}^{\infty} x^{-\beta}(\log x)^{-\alpha} d x .
$$

Then $f(\alpha, \beta, u)$ is bounded below by

$$
\frac{1}{\beta-1} u^{-\beta+1}(\log u)^{-\alpha}-\frac{\alpha}{(\beta-1)^{2}} u^{-\beta+1}(\log u)^{-\alpha-1} .
$$

Likewise, $f(\alpha, \beta, u)$ is bounded above by 


$$
\frac{1}{\beta-1} u^{-\beta+1}(\log u)^{-\alpha}
$$

(b) $\sum_{j=n}^{\infty} j^{-\beta}(\log j)^{-\alpha}=\frac{1}{\beta-1} n^{-\beta+1}(\log n)^{-\alpha}+O\left[n^{-\beta+1}(\log n)^{-\alpha-1}\right]$.

(c) Let $\alpha>1$. Then

$$
\int_{u}^{\infty} x^{-1}(\log x)^{-\alpha} d x=\frac{1}{\alpha-1}(\log u)^{-\alpha+1} .
$$

Proof. Claim (a). Clearly,

$$
f(\alpha, \beta, u)=\frac{1}{\beta-1} u^{-\beta+1}(\log u)^{-\alpha}-\frac{\alpha}{\beta-1} f(\alpha+1, \beta, u) .
$$

In particular,

$$
f(\alpha, \beta, u) \leqq \frac{1}{\beta-1} u^{-\beta+1}(\log u)^{-\alpha} .
$$

Using (5.14) with $\alpha+1$ in place of $\alpha$,

$$
f(\alpha, \beta, u) \geqq \frac{1}{\beta-1} u^{-\beta+1}(\log u)^{-\alpha}-\frac{\alpha}{(\beta-1)^{2}} u^{-\beta+1}(\log u)^{-\alpha-1} .
$$

Claim (b). Clearly, $x^{-\beta}(\log x)^{-\alpha}$ is monotone. So, the sum in (b) is bounded between $f(\alpha, \beta, n)$ and $f(\alpha, \beta, n)+n^{-\beta}(\log n)^{-\alpha}$.

Claim (c). This is clear.

Of course, the estimates in $(5.13 a-b)$ can be developed into asymptotic series.

We now begin to investigate the probability density $q$ defined by (5.3). The following repeated integrals of the tail of $q$ will be useful.

Lemma 5.15. Define $q(j)$ and $b$ by (5.3). For $x>0$, set

$$
\begin{aligned}
& F(x)=\sum_{|j|>x} q(j) \\
& G(x)=\int_{x}^{\infty} F(u) d u \\
& H(x)=\int_{x}^{\infty} G(u) d u .
\end{aligned}
$$

Then, as $x \rightarrow \infty$,
(a)
$F(x)=b x^{-2}(\log x)^{-a}+O\left[x^{-2}(\log x)^{-a-1}\right]$
(c) $G(x)=b x^{-1}(\log x)^{-a}+O\left[x^{-1}(\log x)^{-a-1}\right]$ $H(x)=\frac{b}{a-1}(\log x)^{-a+1}+O\left[(\log x)^{-a}\right]$. 
Proof. This is immediate from (5.13).

Note. Claims (b) and (c) follow from (a). In fact, Theorem (5.9) and the supporting results $(5.17-20-23)$ hold provided the $X$ 's are symmetric, interger-valued, aperiodic and satisfy (5.15a), namely,

$$
P(|X|>x)=b x^{-2}(\log x)^{-a}+O\left[x^{-2}(\log x)^{-a-1}\right]
$$

as $x \rightarrow \infty$. It is the precise bound on the remainder, namely $O\left[x^{-2}(\log x)^{-a-1}\right]$, which enables us to push the calculations through. In this generality, there is no connection between $a$ and $b$, or $\sigma^{2}=$ $\operatorname{Var} X$; the latter is finite by our condition on $P(|X|>x)$.

Let $\theta(t)$ be the characteristic function of $q$. Since $q$ is symmetric,

$$
\theta(t)=\sum_{j} \cos (j t) q(j)
$$

The next lemma gives an approximation for $\theta$. Recall that $\sigma^{2}$ is the variance of $q$ : namely, $\sigma^{2}=\sum_{j} j^{2} q(j)$.

Lemma 5.17. Fix $a>1$. As $t$ tends to 0 ,

$$
\theta(t)=1-\frac{1}{2} \sigma^{2} t^{2}+r(t)
$$

where

$$
\boldsymbol{r}(t)=\frac{b}{a-1} t^{2}\left(\log \frac{1}{|t|}\right)^{-x+1}+O\left[t^{2}\left(\log \frac{1}{|t|}\right)^{-a}\right]
$$

and $b$ is defined in (5.3).

Proof. By symmetry, it is enough to do the case where $t \rightarrow 0^{+}$. Integrating by parts three times gives

$$
\theta(t)=1-\frac{1}{2} \sigma^{2} t^{2}+r(t)
$$

where

$$
r(t)=t^{3} \int_{0}^{\rightarrow \infty} \sin (t x) H(x) d x,
$$

and $H$ is defined in (5.15). Clearly, $H$ is convex and $H(0)<\infty$ because $\sigma^{2}<\infty$. Now (5.7) shows that

$$
t^{2} \frac{1}{2} I_{0} \leqq r(t) \leqq t^{2}\left(I_{0}-\frac{1}{2} I_{1}\right)
$$

with $I_{0}$ and $I_{1}$ defined as in (5.9). To approximate $I_{0}$, break the 
range of integration into two zones $[0, \sqrt{t}]$ and $[\sqrt{t, \pi}]$. For the first zone,

$$
0 \leqq \int_{0}^{\sqrt{t}} H(x / t) \sin x d x<H(0) \int_{0}^{\sqrt{t}} x d x=\frac{1}{2} H(0) t .
$$

For the second zone, use the approximation (5.15c) for $H(x / t)$, along with (5.12). Combining the two zones we have:

$$
I_{0}=\frac{2 b}{a-1}\left(\log \frac{1}{t}\right)^{-a+1}+O\left[\left(\log \frac{1}{t}\right)^{-a}\right] \text {. }
$$

The same estimate for $I_{1}$ can be obtained by the same argument. Substituting into (5.19) completes the proof.

We will use Lemma (5.17) in the following form:

Lemma 5.20. Define $q$ and $b$ by (5.3). Let $\theta(t)$ be the characteristic function of $q$ defined in (5.16). Let $\sigma^{2}$ be the variance of $q$. Then, as $t \rightarrow 0$,

$$
\exp \left(\frac{1}{2} \sigma^{2} t^{2}\right) \theta(t)=1+\gamma(t)+\rho(t)
$$

where

$$
\gamma(t)=\frac{b}{a-1} t^{2}\left(\log \frac{1}{|t|}\right)^{-a+1}
$$

and

$$
\rho(t)=O\left[t^{2}\left(\log \frac{1}{|t|}\right)^{-a}\right]
$$

The next two facts are well known, but are recorded here for ease of reference.

LeMma 5.21. Let $z_{j}$ and $z_{j}^{\prime}$ be complex numbers of absolute value at most $A$. Then

$$
\left|\prod_{j=1}^{n} z_{j}-\prod_{j=1}^{n} z_{j}^{\prime}\right| \leqq A^{n} \sum_{j=1}^{n}\left|z_{j}-z_{j}^{\prime}\right| \text {. }
$$

Lemma 5.22. Let $n$ be a positive integer, and $z$ a complex number. Then

$$
\left|(1+z)^{n}-1-n z\right| \leqq \frac{1}{2} n^{2}|z|^{2} e^{n|z|}
$$

We are now ready to give an approximation for the probability density of $S_{n}$. We will abbreviate the normal density with mean 0 and variance $\sigma^{2}$ by $\varphi_{\sigma}$ : 


$$
\varphi_{\sigma}(x)=\frac{1}{\sigma \sqrt{2 \pi}} \exp \left(-\frac{1}{2} x^{2} / \sigma^{2}\right) .
$$

Also, we introduce the Hermite polynomial:

$$
h_{o}(x)=(x / \sigma)^{2}-1 \text {. }
$$

Proposition 5.23. Fix $a>1$. Let $X_{1}, X_{2}, \cdots$ be independent with common distribution (5.3). Let $S_{n}=X_{1}+\cdots+X_{n}$. Then, as $n \rightarrow \infty$,

$$
\sqrt{n} P\left(S_{n}=j\right)=\varphi_{o}(j / \sqrt{n})\left[1-\varepsilon_{n} h_{\sigma}(j / \sqrt{n})\right]+r_{j n},
$$

where

$$
\begin{gathered}
\sigma^{2}=\operatorname{Var} X_{i}, \\
\varepsilon_{n}=\frac{b}{a-1} /(\log \sqrt{n})^{a-1} \text { with b as in (5.3), }
\end{gathered}
$$

and

$$
\begin{aligned}
d & =\min (a, 2 a-2)>a-1 \\
r_{j n} & =O(\log n)^{-d} \text { uniformly in } j .
\end{aligned}
$$

Note. Similar techniques would give more terms in an Edgeworthlike expansion.

Proof. Our argument is adapted from $\S 16.2$ of Feller [3]. By the Fourier inversion formula,

$$
\varphi_{\sigma}(x)=\frac{1}{2 \pi} \int_{-\infty}^{\infty} \exp (-i t x) \exp \left(-\frac{1}{2} \sigma^{2} t^{2}\right) d t .
$$

Differentiate twice with respect to $x$ :

$$
h_{\sigma}(x) \varphi_{\sigma}(x)=-\frac{1}{2 \pi} \int_{-\infty}^{\infty} \exp (-i t x) t^{2} \exp \left(-\frac{1}{2} \sigma^{2} t^{2}\right) d t
$$

Likewise,

$$
P\left(S_{n}=j\right)=\frac{1}{2 \pi} \int_{-\pi}^{\pi} \exp (-i t j) \theta(t)^{n} d t .
$$

Changing variables,

$$
\sqrt{n} P\left(S_{n}=j\right)=\frac{1}{2 \pi} \int_{-\pi \sqrt{n}}^{\pi \sqrt{n}} \exp (-i t j / \sqrt{n}) \theta(t / \sqrt{n})^{n} d t .
$$

Combining (5.24-25-26), we have

$$
\begin{aligned}
r_{j_{n}} & =\sqrt{n} P\left(S_{n}=j\right)-\varphi_{\sigma}(j / \sqrt{n})+\varepsilon_{n} \varphi_{\sigma}(j / \sqrt{n}) h_{\sigma}(j / \sqrt{n}) \\
& =J_{1}+J_{2}
\end{aligned}
$$


where

$$
J_{1}=\frac{1}{2 \pi} \int_{-\pi \sqrt{ } \bar{n}}^{\pi \sqrt{n}} \exp (-i t j / \sqrt{n}) \psi_{n}(t) d t
$$

with

$$
\psi_{n}(t)=\theta(t / \sqrt{n})^{n}-\left(1+\varepsilon_{n} t^{2}\right) \exp \left(-\frac{1}{2} \sigma^{2} t^{2}\right)
$$

and

$$
J_{2}=-\frac{1}{2 \pi} \int_{|t|>\pi \sqrt{n}} \exp (-i t j / \sqrt{n})\left(1+\varepsilon_{n} t^{2}\right) \exp \left(-\frac{1}{2} \sigma^{2} t^{2}\right) d t .
$$

Clearly,

$$
\left|J_{2}\right| \leqq \frac{1}{2 \pi} \int_{|t|>\pi \sqrt{n}}\left(1+\varepsilon_{n} t^{2}\right) \exp \left(-\frac{1}{2} \sigma^{2} t^{2}\right) d t,
$$

so $J_{2}$ is negligible.

Likewise,

$$
\left|J_{1}\right| \leqq \frac{1}{2 \pi} \int_{-\pi \sqrt{n}}^{\pi \sqrt{n}}\left|\psi_{n}(t)\right| d t
$$

We split the interval of integration in (5.28) into three sub-intervals:

$$
\begin{aligned}
& R_{1}, \text { the set of } t \text { with }|t| \leqq n^{1 / 4} \\
& R_{2}, \text { the set of } t \text { with } n^{1 / 4}<|t| \leqq \delta n^{1 / 2} \\
& R_{3} \text {, the set of } t \text { with } \delta n^{1 / 2}<|t| \leqq \pi n^{1 / 2}
\end{aligned}
$$

We will choose $\delta>0$ later.

Consider $R_{3}$. The distribution $q(j)$ of (5.3) is aperiodic, so $\sup _{|t|>o}|\theta(t)|=\lambda<1$. Of course

$$
\left|\psi_{n}(t)\right| \leqq \theta(t / \sqrt{n})^{n}+\left(1+\varepsilon_{n} t^{2}\right) \exp \left(-\frac{1}{2} \sigma^{2} t^{2}\right) .
$$

So the contribution from $R_{3}$ to the integral in (5.28) is at most

$$
\lambda^{n} \sqrt{n}+\int_{|t|>\bar{\delta} \sqrt{n}}\left(1+\varepsilon_{n} t^{2}\right) \exp \left(-\frac{1}{2} \sigma^{2} t^{2}\right) d t .
$$

This is negligible.

Next consider $R_{2}$. Using (5.17), we may choose $\delta>0$ so small that

$$
|\theta(u)|<1-\frac{1}{4} \sigma^{2} u^{2}<\exp \left(-\frac{1}{4} \sigma^{2} u^{2}\right)
$$

for $|u|<\delta$. This fixes the $\delta$ defining $R_{2}$. In particular, 


$$
|\theta(t / \sqrt{n})|^{n}<\exp \left(-\frac{1}{4} \sigma^{2} t^{2}\right)
$$

for all $t$ in $R_{2}$. As a result, the contribution from $R_{2}$ to the integral in (5.28) is negligible.

We now show that the contribution from $R_{1}$ to the integral in $(5.28)$ is $O(\log n)^{-a}+O(\log n)^{2-2 a}$. Let

$$
\beta_{n}(t)=\exp \left(\frac{1}{2} \sigma^{2} t^{2} / n\right) \theta(t / \sqrt{n})-1
$$

Recall the definition of $\psi_{n}$ from (5.27). Clearly

$$
\psi_{n}(t)=\left\{\left[1+\beta_{n}(t)\right]^{n}-1-\varepsilon_{n} t^{2}\right\} \exp \left(-\frac{1}{2} \sigma^{2} t^{2}\right)
$$

Recall $\gamma$ and $\rho$ from (5.20). As that result shows,

$$
\beta_{n}(t)=\gamma(t / \sqrt{n})+\rho(t / \sqrt{n}) .
$$

Use (5.20) again: there are positive, finite constants $K_{i}$ such that for all $t$ in $R_{1}$

$$
|n \rho(t / \sqrt{n})| \leqq K_{1} t^{2} /(\log n)^{a}
$$

and then

$$
\left|n \beta_{n}(t)\right| \leqq K_{2} t^{2} /(\log n)^{a-1}
$$

so

$$
\left|n \beta_{n}(t)\right| \leqq \frac{1}{4} \sigma^{2} t^{2} \text { for all large } n
$$

We are estimating

$$
J_{1}^{*}=\frac{1}{2 \pi} \int_{R_{1}}\left|\psi_{n}(t)\right| d t
$$

Using (5.29), (5.30), and the triangle inequality, we have

$$
J_{1}^{*} \leqq J_{a}+J_{b}+J_{c}
$$

where

$$
\begin{aligned}
& J_{a}=\frac{1}{2 \pi} \int_{R_{1}}|n \rho(t / \sqrt{n})| \exp \left(-\frac{1}{2} \sigma^{2} t^{2}\right) d t \\
& J_{b}=\frac{1}{2 \pi} \int_{R_{1}}\left|\left[1+\beta_{n}(t)\right]^{n}-\left[1+n \beta_{n}(t)\right]\right| \exp \left(-\frac{1}{2} \sigma^{2} t^{2}\right) d t \\
& J_{c}=\frac{1}{2 \pi} \int_{R_{1}}\left|n \gamma(t / \sqrt{n})-\varepsilon_{n} t^{2}\right| \exp \left(-\frac{1}{2} \sigma^{2} t^{2}\right) d t
\end{aligned}
$$

We estimate $J_{a}$, using (5.31): 


$$
\begin{aligned}
J_{a} & \leqq \frac{1}{2 \pi}(\log n)^{-a} \int t^{2} \exp \left(-\frac{1}{2} \sigma^{2} t^{2}\right) d t \\
& =O(\log n)^{-a} .
\end{aligned}
$$

We estimate $J_{b}$, using (5.22):

$$
\left|\left[1+\beta_{n}(t)\right]^{n}-1-n \beta_{n}(t)\right| \leqq \frac{1}{2}\left|n \beta_{n}(t)\right|^{2} \exp \left(\left|n \beta_{n}(t)\right|\right) .
$$

In view of (5.32-33), this bound is at most

$$
\frac{1}{2} K_{2}^{2}(\log n)^{2-2 a} t^{4} \exp \left(\frac{1}{4} \sigma^{2} t^{2}\right) \text {. }
$$

So

$$
\begin{aligned}
J_{b} & \leqq \frac{1}{4 \pi} K_{2}^{2}(\log n)^{2-2 a} \int t^{4} \exp \left(-\frac{1}{4} \sigma^{2} t^{2}\right) d t \\
& =O(\log n)^{2-2 a} .
\end{aligned}
$$

Finally, we estimate $J_{c}$. This is more delicate, and it is convenient to treat two zones separately. Let

$$
\begin{aligned}
& R_{1}^{+} \text {be the set of } t \text { with } 1<|t| \leqq n^{1 / 4} \\
& R_{1}^{-} \text {be the set of } t \text { with }|t| \leqq 1
\end{aligned}
$$

So $R_{1}=R_{1}^{+} \cup R_{1}^{-}$. Using the definition of $\gamma$ in (5.20), and the definition of $\varepsilon_{n}$ in (5.33), the contribution to $J_{c}$ from $R_{1}^{+}$is

$$
\frac{1}{2 \pi} \frac{b}{a-1} \frac{1}{\left(\log \sqrt{n)^{a-1}}\right.}
$$

times

$$
\int_{R_{1}^{+}}\left|\left(1-\frac{\log |t|}{\log \sqrt{n}}\right)^{1-a}-1\right| t^{2} \exp \left(-\frac{1}{2} \sigma^{2} t^{2}\right) d t .
$$

The integrand in (5.35) can be estimated by (5.11c), because $|t|<$ $n^{1 / 4}$ in $R_{1}$, so $\log |t| / \log \sqrt{n}<1 / 2$ in $R_{1}$. Hence (5.35) is at most

$$
\frac{K}{\log \sqrt{n}} \int_{|t|>1}(\log |t|) t^{2} \exp \left(-\frac{1}{2} \sigma^{2} t^{2}\right) d t \text {. }
$$

This is $O(\log n)^{-1}$. Thus, the contribution to $J_{c}$ from $R_{1}^{+}$is at most $O(\log n)^{-a}$. Likewise, the contribution to $J_{c}$ from $R_{1}^{-}$is the factor (5.34) times

$$
\int_{R_{1}^{-}}\left|\left[1+\frac{\log (1 /|t|)}{\log \sqrt{n}}\right]^{1-a}-1\right| t^{2} \exp \left(-\frac{1}{2} \sigma^{2} t^{2}\right) d t
$$

By $(5.11 b)$, this is at most 


$$
\begin{aligned}
& \frac{a-1}{\log \sqrt{n}} \int_{-1}^{1}\left(\log \frac{1}{|t|}\right) t^{2} \exp \left(-\frac{1}{2} \sigma^{2} t^{2}\right) d t \\
& \quad+2^{a} \frac{a(a-1)}{(\log \sqrt{n})^{2}} \int_{-1}^{1}\left(\log \frac{1}{|t|}\right)^{2} t^{2} \exp \left(-\frac{1}{2} \sigma^{2} t^{2}\right) d t .
\end{aligned}
$$

This too is $O(\log n)^{-1}$. Thus, the contribution to $J_{c}$ from $R_{1}^{-}$is also $O(\log n)^{-a}$.

We are now in position to finish the proof of (5.4). The argument is very similar to the proof of Theorem (1.8) and we just indicate the changes.

Proof of 5.4. The bounds on the maximum deviation in zones IV, III, and II, as given in (4.1), (4.3), and (4.5) go through without change. The estimates used in (4.10) are valid as stated, except that $(4.10 \mathrm{~b})$ becomes:

$$
\begin{aligned}
p_{j}= & P\left(S_{n}=j\right)=\frac{1}{\sigma \sqrt{2 \pi n}} \pi_{j n}, \text { where } \\
\pi_{j_{n}}= & 1-\left[1+O\left(\delta^{2}\right)\right] j^{2} /\left(2 \sigma^{2} n\right)+\frac{b}{a-1}(\log \sqrt{n})^{1-a} \\
& +O\left((\log n)^{2-2 a}\right)
\end{aligned}
$$

uniformly in $j \in$ zone I; the " $O\left(\delta^{2}\right)$ " is uniform in $j$ and $n$. This follows from (5.23). Indeed, since $j$ is confined to zone I, $j^{2} / n^{2}=$ $O\left(\delta^{2}\right)$, and

$$
\exp \left[-j^{2} /\left(2 \sigma^{2} n\right)\right]=1-\left[1+O\left(\delta^{2}\right)\right] j^{2} /\left(2 \sigma^{2} n\right) .
$$

We must now multiply by

$$
1-\frac{b}{a-1}(\log \sqrt{n})^{1-a}\left(\frac{j^{2}}{2 \sigma^{2} n}-1\right) .
$$

We get

$$
1-\left[1+O\left(\delta^{2}\right)\right] j^{2} / 2 \sigma^{2} n+\frac{b}{a-1}(\log \sqrt{n})^{a-1}+T
$$

where

$$
\begin{aligned}
T & =\frac{b}{a-1}(\log \sqrt{n})^{1-a} \frac{j^{2}}{2 \sigma^{2} n}\left\{1+\left[1+O\left(\delta^{2}\right)\right]\left(\frac{j^{2}}{2 \sigma^{2} n}-1\right)\right\} \\
& =o(1) \cdot \frac{j^{2}}{2 \sigma^{2} n}
\end{aligned}
$$

because $a>1$; and $T$ can be merged into the lead term of $\pi_{j n}$. Since $a \leqq 2$, 


$$
d=\min (a, 2 a-2)=2 a-2,
$$

accounting for the error term $O(\log n)^{2-2 a}$ in $\pi_{j_{n}}$. Instead of (4.10c), we must estimate

$$
\begin{aligned}
1 / \pi_{n j} & =1+\left[1+O\left(\delta^{2}\right)\right] j^{2} /\left(2 \sigma^{2} n\right) \\
& -\frac{b}{a-1}(\log \sqrt{n})^{1-a}+O(\log n)^{2-2 a},
\end{aligned}
$$

the higher powers of the lead term merging into the $O\left(\delta^{2}\right)$, because for $j \in$ zone I,

$$
\left(j^{2} / 2 \sigma^{2} n\right)^{2}=O\left(\delta^{2}\right) \cdot j^{2} / 2 \sigma^{2} n .
$$

Lemmas (4.16-17) go through as stated, with $z_{n}(x)$ replaced by $z_{n}(x, a)$. In (4.18a), the bound on $k_{j}^{\prime} p_{j}^{\prime}$ is

$$
\begin{aligned}
& \frac{k}{\sigma \sqrt{2 \pi n}}\left\{1-\left[1+O\left(\delta^{2}\right)\right] \frac{j^{2}}{2 \sigma^{2} n}+\frac{b}{a-1} \frac{1}{(\log \sqrt{n})^{1-a}}\right. \\
& \left.+O(\log n)^{2-2 a}\right\}\left[1+o\left(\frac{1}{\log n}\right)\right] .
\end{aligned}
$$

In $(4.18 \mathrm{~b}), z_{n}(x)$ should be replaced by $z_{n}(x, a)$. There is no problem with (4.18c). Remarkably enough, the bound on $h^{2} / k_{j}^{\prime} p_{j}^{\prime}$ in $(4.18 \mathrm{~d})$ goes through unchanged, with $\mu=0$, and some different $b_{\delta}=0\left(\delta^{2}\right)$. It is here that we use the condition $a>1.5$, as well as the definition of the constant $c$ in (5.4). In essence, $h^{2}$ and $k_{j}^{\prime} p_{j}^{\prime}$ have the same lead factor $k / \sigma \sqrt{2 \pi n}$, which cancels in $h^{2} / k_{j}^{\prime} p_{j}^{\prime}$, leaving the product $U V W$, where

$$
\begin{aligned}
& U=\log n+c(\log n)^{2-a}-2 \log \log n+x \\
& V=1+\left[1+O\left(\delta^{2}\right)\right] \frac{j^{2}}{2 \sigma^{2} n}-\frac{b}{a-1} \frac{1}{(\log \sqrt{n})^{a-1}}+O(\log n)^{2-2 a} \\
& W=1+o(1 / \log n) .
\end{aligned}
$$

The factor $W$ can be ignored. In multiplying out $U V$, the term

$$
\log n \cdot \frac{b}{a-1} \frac{1}{(\log \sqrt{n})^{a-1}}=-2^{a-1} \frac{b}{a-1}(\log n)^{2-a}
$$

cancels the term

$$
c(\log n)^{2-a} \cdot 1,
$$

because $c$ was chosen to be $2^{a-1} b /(a-1)$. Furthermore,

$$
U \cdot O(\log n)^{2-2 a}=o(1),
$$

because we imposed the condition $a>1.5$, so $3-2 a<0$. 
The remaining arguments used in (1.8) go through with only minor changes.

Finally, we indicate why Theorem (1.8) continues to hold when the third-moment condition (1.2) is replaced by the log-moment condition (5.1). Let

$$
\begin{aligned}
F(x) & =P\left(X_{1}>x\right) \text { for } \quad x \geqq 0 \\
& =P\left(X_{1}<x\right) \text { for } \quad x<0 .
\end{aligned}
$$

By Chebychev's inequality,

$$
F(x)=O\left[x^{2}(\log |x|)^{-2-\grave{o}}\right] \text { as } x \longrightarrow \pm \infty .
$$

Let

$$
\begin{aligned}
G(x) & =\int_{x}^{\infty} F(u) d u \text { for } x \geqq 0 \\
& =\int_{-\infty}^{x} F(u) d u \text { for } x<0 .
\end{aligned}
$$

Using (5.13),

$$
G(x)=O\left[|x|^{-1}(\log |x|)^{-2-\hat{o}}\right] \text { as } x \longrightarrow \pm \infty .
$$

Let

$$
\begin{aligned}
H(x) & =\int_{x}^{\infty} G(u) d u \text { for } x>0 \\
& =\int_{-\infty}^{x} G(u) d u \text { for } x<0 .
\end{aligned}
$$

Using (5.13) again,

$$
H(x)=O\left[(\log |x|)^{-1-i}\right] \text { as } x \longrightarrow \pm \infty .
$$

Now, if $\theta(t)$ is the characteristic function of $X_{1}$, and $\mu=E\left(X_{1}\right)$, we can argue as in (5.17) to show that

$$
\theta(t)=1+i \mu t-\frac{1}{2} E\left(X_{1}^{2}\right) t^{2}+r(t)
$$

where as $t \rightarrow 0$,

$$
r(t)=O\left[|t|^{2}\left(\log \frac{1}{|t|}\right)^{-1-o}\right\rceil
$$

Consequently, by an argument like the one used for (5.23) but without the Hermite polynomial,

$$
\sqrt{n} P\left(S_{n}=j\right)=\varphi_{o}[j-n \mu]+O(\log n)^{-1-\grave{o}},
$$


where $\sigma^{2}=\operatorname{Var} X_{1}$. The approximation $(5.40)$ can be used in place of the local central limit Theorem (3.27) in proving (1.8). An approximation similar to $(5.40)$ based on fractional moments (rather than log-moments) is given by Ibragimov [7].

\section{REFERENCES}

1. H. Cramer, On asymptotic expansions for sums of independent random variables with a limiting stable distribution, Sankhya, 25 (1963), Series A, 13-24.

2. P. Diaconis and D. Freedman, On the mode of an empirical histogram for sums, Pacific J. Math., 100 (1982d), 373-385.

3. W. Feller, An Introduction to Probability Theory and Its Applications, Vol. II, (1971), 2nd Ed., Wiley, New York.

4. D. Freedman, Another note on the Borel-Cantelli lemma and the strong law with the Poisson approximation as a by-product, Ann. Prob., 1 (1973), 910-925.

5. D. Freedman, A central limit theorem for empirical histograms, Zeitschrift für Wahrscheinlichkeitsthëories, 41 (1977), 1-11.

6. D. Freedman, R. Pisiani, and R. Purves, Statistics, W. W. Norton, (1978), New York.

7. I. A. Ibragimov, On the accuracy of approximation to the distribution functions of sums of independent variables, Th. Prob. and its Applications II, (1966), 559-579.

8. A. Kolchin, B. Sevastyanov, and C. Chistyakov, Random Allocations, (1978), Wiley, New York.

9. C. L. Mallows, An inequality involving multinomial Probabilities, Biometrika, 55 (1968), 422-424.

10. V. V. Petrov, Sums of Independent Random Variables, (1975), Springer, New York.

11. E. J. G. Pitman, On the behavior of the characteristic function of a probability distribution in the neighborhood of the origin, J. Austral. Math. Soc., 8 (1968), 423-443.

12. K. Takeuchi, and M. Akahira, On Gram-Charlier-Edgeworth type expansions of the sums of random variables (I), Rep. Univ. Electro-Comm., 27 (1976), 95-115.

13. - On Gram-Charlier-Edgeworth type expansions of the sums of random variables (II), Rep. Univ. Electro-Comm., 27 (1976), 117-213.

Received January 7, 1981. Research by the first author was partially supported by N00014-76-C-0472. Research by the second author was partially supported by NSF Grant MCS-77-01665.

Department of Statistics

STANFORD UNIVERSITY

STANFORD, CA 94305

AND

Department of Statistics

UNIVERSITY OF CALIFORNIA

Berkeley, CA 94720 



\section{PACIFIC JOURNAL OF MATHEMATICS}

\section{EDITORS}

DONALD BABBITT (Managing Editor)

University of California

Los Angeles, CA 90024

Hugo RossI

University of Utah

Salt Lake City, UT 84112

C. C. Moore and Arthur Agus

University of California

Berkeley, CA 94720
J. DugundJI

Department of Mathematics

University of Southern California

Los Angeles, CA 90007

R. FinN and J. MiLgRAM

Stanford University

Stanford, CA 94305

\section{ASSOCIATE EDITORS}
R. ARENS
E. F. BECKENBACH
B. H. NeumanN
F. WOLF
K. YoSHIDA

\section{SUPPORTING INSTITUTIONS}

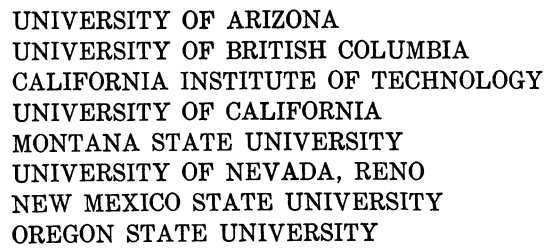

UNIVERSITY OF ARIZONA

UNIVERSITY OF BRITISH COLUMBIA CALIFORNIA INSTITUTE OF TECHNOLOGY UNIVERSITY OF CALIFORNIA MONTANA STATE UNIVERSITY

UNIVERSITY OF NEVADA, RENO NEW MEXICO STATE UNIVERSITY OREGON STATE UNIVERSITY

\author{
UNIVERSITY OF OREGON \\ UNIVERSITY OF SOUTHERN CALIFORNIA \\ STANFORD UNIVERSITY \\ UNIVERSITY OF AAWAII \\ UNIVERSITY OF TOKYO \\ UNIVERSITY OF UTAH \\ WASHINGTON STATE UNIVERSITY \\ UNIVERSITY OF WASHINGTON
}

The Supporting Institutions listed above contribute to the cost of publication of this Journal, but they are not owners or publishers and have no responsibility for its content or policies,

Mathematical parers intended for publication in the Pacific Journal of Mathematics should be in typed form or offset-reproduced, (not dittoed), double spaced with large margins. Please do not use built up fractions in the text of the manuscript. However, you may use them in the displayed equations. Underline Greek letters in red, German in green, and script in blue. The first paragraph or two must be capable of being used separately as a synopsis of the entire paper. Please propose a heading for the odd unmbered pages of less than 35 characters. Manuscripts, in triplicate, may be sent to any one of the editors. Please classify according to the scheme of Math. Reviews, Index to Vol. 39. Supply name and address of author to whom proofs should be sent. All other communications should be addressed to the managing editor, or Elaine Barth, University of California, Los Angeles, California, 90024 .

50 reprints to each author are provided free for each article, only if page charges have been substantially paid. Additional copies may be obtained at cost in multiples of 50 .

The Pacific Journal of Mathematics is issued monthly as of January 1966, Regular subscription rate: $\$ 114.00$ a year (6 Vol., 12 issues). Special rate: $\$ 57.00$ a year to individual members of supporting institution.

Subscriptions, orders for numbers issued in the last three calendar years, and changes of address shoud be sent to Pacific Journal of Mathematics, P.O. Box 969, Carmel Valley, CA 93924, U.S.A. Old back numbers obtainable from Kraus Periodicals Co., Route 100, Millwood, NY 10546.

\section{PUBLISHED BY PACIFIC JOURNAL OF MATHEMATICS, A NON-PROFIT CORPORATION}

Printed at Kokusai Bunken Insatsusha (International Academic Printing Co., Ltd.). 8-8, 3-chome, Takadanobaba, Shinjuku-ku, Tokyo 160, Japan.

Copyright (C) 1982 by Pacific Journal of Mathematics Manufactured and first issued in Japan 


\section{Pacific Journal of Mathematics}

Vol. 100, No. $2 \quad$ October, 1982

Kenneth F. Andersen, On the transformation of Fourier coefficients of

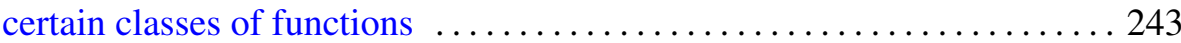

Steven Albert Bleiler, Realizing concordant polynomials with prime knots

Reinhard Bürger, Functions of translation type and solid Banach spaces of functions

Ulrich Daepp, The saturation of $k$-analytic rings and topological equivalence of associated analytic set germs .................. 271

Persi W. Diaconis and David Amiel Freedman, On the maximum difference between the empirical and expected histograms for sums . . . 287

David Amiel Freedman, On the maximum of scaled multinomial

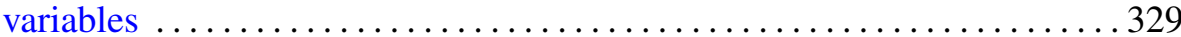

Persi W. Diaconis and David Amiel Freedman, On the difference between the empirical histogram and the normal curve, for sums. II ......... 359

Persi W. Diaconis and David Amiel Freedman, On the mode of an

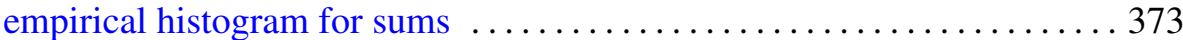

Jutta Hausen, Supplemented modules over Dedekind domains 387

Elyahu Katz, A moduli representation for the classification of twisted tensor

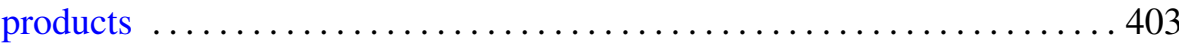

H. C. Madhekar and N. K. Thakare, Biorthogonal polynomials suggested by the Jacobi polynomials

Ted R. Pettis, Collections of covers of metric spaces 425

Ryōtarō Satō, Maximal functions for a semiflow in an infinite measure space

Michael Jay Stob, Invariance of properties under automorphisms of the lattice of recursively enumerable sets 